\title{
On a Theorem of Bandt and Wang and Its Extension to $p$-tiles
}

\author{
Benoit Loridant · Jun Luo
}

Received: 3 October 2007 / Revised: 11 July 2008 / Accepted: 21 November 2008 /

Published online: 6 January 2009

(C) Springer Science+Business Media, LLC 2008

\begin{abstract}
We study tilings of the plane by a single prototile with respect to the lattice and to the crystallographic group $p 2$. We are interested in the connection between the neighbors of a tile in the tiling and its topology. We show that lattice and $p 2$-tiles always have at least six neighbors. We characterize self-affine tiles that are homeomorphic to a disk in a rather easy way by the set and number of neighbors of the central tile in the tiling. This extends the work of Bandt and Wang devoted to lattice self-affine disk-like tiles of the plane.
\end{abstract}

Keywords Crystallographic reptiles · Tiling · Homeomorphy to a disk

\section{Introduction}

Let $\Gamma$ be a group of isometries on $\mathbb{R}^{2}$. We consider plane compact tiles $T$ that provide a tiling $\{\gamma(T), \gamma \in \Gamma\}$ of the plane and have the replication property

$$
g(T)=\bigcup_{\delta \in \mathcal{D}} \delta(T)
$$

for some expanding map $g$ and finite digit set $\mathcal{D} \subset \Gamma$.

The first author was supported by the Austria-Ungary Project Nr 67öu1, Austrian Science Foundation (FWF), projects S9604 and S9610, that is part of the Austrian National Research Network "Analytic combinatorics and Probabilistic Number Theory"; the second author was supported by the National Natural Science Foundation of China, project 10601069.

B. Loridant $(\bowtie)$

Department of Mathematics, Faculty of Science, Niigata University, Ikarashi 2 8050, Niigata, 950 2181, Japan

e-mail: loridant@math.sc.niigata-u.ac.jp

J. Luo

School of Mathematics and Computational Science, Sun Yat-Sen University, Guangzhou 512075, China

e-mail: luojun3@mail.sysu.edu.cn 
In recent works on the topology of crystallographic reptiles (or crystiles for short), Loridant et al. obtained criteria in order to decide whether a given tile $T$ as above is homeomorphic to a closed disk (see [14, 15]). The disk-likeness of a tile $T$ is strongly related to the number of its neighbors (tiles other than $T$ intersecting $T$ ) and their configuration in the tiling. We are interested in easily checkable criteria of disk-likeness for two classes of crystiles corresponding to two particular choices of the group $\Gamma$. Bandt and Wang gave in [4] a criterion for the class of self-affine lattice tiles that tile the plane by their translates ( $\Gamma$ is thus isomorphic to $\mathbb{Z}^{2}$ ). In this case, disk-likeness happens only if $T$ has six or eight neighbors and eventually depends on the shape of the digit set $\mathcal{D}$. Our aim is to extend this characterization to the class of $p 2$-crystiles that tile the plane in the following way: the union of $T$ with its image under a $\pi$-rotation tiles the plane by its translates. The technique of our proof makes use of graphs associated to the tilings and also allows us to give a new complete proof of Bandt and Wang's criterion, which concerned the lattice case. Moreover, on the way to the proof, we will see that lattice and $p 2$-tiles (not necessarily with a replication property) always have at least six neighbors, whose configuration is fixed if the number of neighbors is exactly six. Let us recall some definitions and facts about tilings and crystiles.

A tiling of $\mathbb{R}^{n}$ is a cover of the space by nonoverlapping sets, i.e., such that the interior of two distinct sets of the cover are disjoint. Some particular tilings use a single tile $T$ with $\overline{T^{o}}=T$ and a family $\Gamma$ of isometries of $\mathbb{R}^{n}$ such that

$$
\mathbb{R}^{n}=\bigcup_{\gamma \in \Gamma} \gamma(T) .
$$

$T$ is a prototile of the tiling. If $\Gamma$ contains id, the identity map of $\mathbb{R}^{n}, T$ is also called the central tile of the tiling. Two distinct tiles are said to be neighbors if they intersect each other. Two neighbors are adjacent neighbors if the interior of their union contains a point of their intersection. The neighbor set of $T$ is then given by

$$
\mathcal{S}=\{\gamma \in \Gamma-\{\operatorname{id}\}, \gamma(T) \cap T \neq \emptyset\} .
$$

It is symmetric and it generates $\Gamma(\Gamma=\langle\mathcal{S}\rangle)$. The adjacent neighbor set $\mathcal{A} \subset \mathcal{S}$ is defined similarly as the set of adjacent neighbors of the identity

$$
\mathcal{A}=\left\{\gamma \in \mathcal{S}, T \cap \gamma(T) \cap(T \cup \gamma(T))^{o} \neq \emptyset\right\} .
$$

The neighbor (resp. adjacent neighbor) set of a tile $\gamma(T)(\gamma \in \Gamma)$ is equal to $\gamma \mathcal{S}$ (resp. $\gamma \mathcal{A}$ ). The tiles considered in this paper will be compact and the tilings locally finite, i.e., every compact set intersects finitely many tiles of the tiling. Thus $\mathcal{S}$ is here always a finite set.

We will deal with families $\Gamma$ of isometries that are crystallographic groups in dimension 2, i.e., discrete cocompact subgroups $\Gamma$ of the group $\operatorname{Isom}\left(\mathbb{R}^{2}\right)$ of all isometries on $\mathbb{R}^{2}$ with respect to some metric. By a theorem of Bieberbach (see [5]), a crystallographic group $\Gamma$ in dimension 2 contains a group $\Lambda$ of translations isomorphic to the lattice $\mathbb{Z}^{2}$, and the quotient group $\Gamma / \Lambda$, called point group, is finite. There are 17 nonisomorphic such groups. However, in this paper, we will mainly consider either 
lattice groups (i.e., for which the point group only contains the class of the identity map of $\mathbb{R}^{2}$ ), or the following crystallographic $p 2$-groups.

Definition 1.1 Let $u(x, y)=(x+1, y), v(x, y)=(x, y+1), r(x, y)=(-x,-y)$. Then a p2-group is a group of isometries of $\mathbb{R}^{2}$ isomorphic to the subgroup of Isom $\left(\mathbb{R}^{2}\right)$ generated by the translations $u, v$ and the $\pi$-rotation $r$.

Eventually, we will always consider that the groups $\Gamma$ involved in the tilings below are the exact symmetry groups of the tilings (see [9]), and call the corresponding tilings $\Gamma$-tilings, the central tile a $\Gamma$-tile. Thus if $\Gamma$ is a lattice group, the tiling is a lattice tiling, if it is a $p 2$-group, a $p 2$-tiling, and similarly for the tiles.

Our main results will concern a class of self-affine tiles constructed in the following way (we refer the reader to $[8,14]$ for further information about these tiles).

Definition 1.2 A crystallographic reptile (or simply crystile) with respect to a crystallographic group $\Gamma$ is a compact nonempty set $T \subset \mathbb{R}^{n}$ with the following properties:

- The family $\{\gamma(T): \gamma \in \Gamma\}$ is a tiling of $\mathbb{R}^{n}$.

- There is an expanding affine map $g: \mathbb{R}^{n} \rightarrow \mathbb{R}^{n}$ such that $g \circ \Gamma \circ g^{-1} \subset \Gamma$ and there exists a finite collection $\mathcal{D} \subset \Gamma$ called digit set such that

$$
g(T)=\bigcup_{\delta \in \mathcal{D}} \delta(T)
$$

We recall that an expanding affine map $g$ has the form $g(x)=A x+b$, where $b \in \mathbb{R}^{n}$ and $A$ is an $n \times n$-matrix whose eigenvalues all have modulus greater than 1 . Hence the compact nonempty set $T$ in the above definition is uniquely defined by (1.1). This is easily seen by a fixed-point argument (cf. [11]).

In the remark below, $T$ is a crystile with neighbor set $\mathcal{S}$, and we use the notation of Definition 1.2.

\section{Remark 1.3}

1. (See [8]) Without loss of generality, one can assume that the digit set $\mathcal{D}$ contains id. Moreover, for $T$ to cover the space by its copies $\{\gamma(T), \gamma \in \Gamma\}$, the set $\mathcal{D}$ must be a complete set of right coset representatives of $\Gamma / g \Gamma g^{-1}$.

2. By definition, the expanded shape $g(T)$ is the union of $\delta(T)$ with $\delta$ running over $\mathcal{D}$. Thus, for each $\gamma \in \Gamma$, there is a uniquely determined collection $\mathcal{D}_{\gamma} \subset \Gamma$ such that $g \gamma(T)$ is the union of all the tiles $\delta(T)$ with $\delta$ running over $\mathcal{D}_{\gamma}$; more precisely, we have $\mathcal{D}_{\gamma}=g \gamma g^{-1} \mathcal{D}$.

We mention that algorithms were given in [14] in order to compute the neighbor and adjacent neighbor sets $\mathcal{S}$ and $\mathcal{A}$ of a cystallographic reptile from the only knowledge of $\Gamma, \mathcal{D}$, and $g$. 


\section{Results}

We present our main results and illustrate some of them.

Concerning the least number of neighbors of a tile in a tiling, we will prove the following:

Theorem 2.1 In a lattice tiling or a p2-tiling of the plane using a compact prototile, each tile has at least six neighbors.

The lattice case was already obtained in [4, Theorem 4.2], where the connectivity of the tiles was used. We remove this assumption of connectivity: our proof just relies on the existence of triple points, i.e., points where at least three distinct tiles meet.

The aim of Bandt and Wang in [4] was the following criterion of disk-likeness for plane tiles defined as in Definition 1.2 in the case that $\Gamma$ is a plane lattice. First we need a definition.

Definition 2.2 If $\mathcal{D}$ and $\mathcal{F}$ are two sets of isometries in $\mathbb{R}^{2}$, we say that $\mathcal{D}$ is $\mathcal{F}$-connected iff for every disjoint pair $\left(d, d^{\prime}\right)$ of elements in $\mathcal{D}$, there exist $n \geq 1$ and elements $d=: d_{0}, d_{1}, \ldots, d_{n-1}, d_{n}:=d^{\prime}$ of $\mathcal{D}$ such that $d_{i}^{-1} d_{i+1} \in \mathcal{F}$ for $i=$ $0, \ldots, n-1$.

This notion of set-connectedness is related to the connectedness of tiles: it was shown in [12] that a necessary and sufficient condition for a crystile as in Definition 1.2 to be connected is that the digit set is $\mathcal{S}$-connected.

Proposition 2.3 [4] Let $T$ be a self-affine lattice plane tile with digit set $\mathcal{D}$ :

(1) Suppose that the neighbor set $\mathcal{S}$ of $T$ has not more than six elements. Then $T$ is disk-like iff $\mathcal{D}$ is $\mathcal{S}$-connected.

(2) Suppose that the neighbor set $\mathcal{S}$ of $T$ has eight elements $\left\{a^{ \pm 1}, b^{ \pm 1},(a b)^{ \pm 1}\right.$, $\left.\left(a b^{-1}\right)^{ \pm 1}\right\}$, where $a$ and $b$ denote two independent translations. Then $T$ is disklike iff $\mathcal{D}$ is $\left\{a^{ \pm 1}, b^{ \pm 1}\right\}$-connected.

We will give a new proof of this result and extend it to the $p 2$-groups as follows.

Theorem 2.4 Let $T$ be a crystile that tiles the plane by a p2-group. Let $\mathcal{D}$ be the corresponding digit set:

(1) Suppose that the neighbor set $\mathcal{S}$ of $T$ has six elements. Then $T$ is disk-like iff $\mathcal{D}$ is $\mathcal{S}$-connected.

(2) Suppose that the neighbor set $\mathcal{S}$ of $T$ has seven elements

$$
\left\{b^{ \pm 1}, c, b c, a^{-1} c, a^{-1} b c, a^{-1} b^{-1} c\right\}
$$

where $a, b$ are translations, and $c$ is $a \pi$-rotation.

Then $T$ is disk-like iff $\mathcal{D}$ is $\left\{b^{ \pm 1}, c, b c, a^{-1} c\right\}$-connected. 
(3) Suppose that the neighbor set $\mathcal{S}$ of $T$ has eight elements

$$
\begin{aligned}
& \left\{b^{ \pm 1}, c, a^{-1} c, b c, b^{-1} c, a^{-1} b c, a^{-1} b^{-1} c\right\} \\
& \quad\left(\text { resp. } \quad\left\{c, b c, a c, a b^{-1} c, b^{ \pm 1},\left(a^{-1} b\right)^{ \pm 1}\right\}\right),
\end{aligned}
$$

where $a, b$ are translations, and $c$ is $a \pi$-rotation.

Then $T$ is disk-like iff $\mathcal{D}$ is $\left\{b^{ \pm 1}, c, a^{-1} c\right\}$-(resp. $\left\{c, b c, a c, a b^{-1} c\right\}$-)connected.

(4) Suppose that the neighbor set $\mathcal{S}$ of $T$ has twelve elements

$$
\left\{c, a^{-1} c, b c, a b c, a^{-1} b c, a^{-1} b^{-1} c, a^{ \pm 1}, b^{ \pm 1},(a b)^{ \pm 1}\right\},
$$

where $a, b$ are translations, and $c$ is a $\pi$-rotation.

Then $T$ is disk-like iff $\mathcal{D}$ is $\left\{c, a^{-1} c, b c\right\}$-connected.

Remark 2.5 (To Proposition 2.3 and Theorem 2.4)

1. According to Grünbaum and Shephard's classification of isohedral tilings (see [9, Sect. 6.2, p. $285 \mathrm{ff}]$ ), the mentioned cases are the only ones for disk-like lattice and p2-tiles in the plane.

2. In each item of these statements, the set with respect to which the digit set is connected reveals to be exactly the set of adjacent neighbors of the central tile. Indeed, it corresponds to the set of adjacent neighbors of the involved disk-like tile in Grünbaum and Shephard's classification (see [9]).

3. Propositions 4.1 and 4.4 will give the exact shape of the neighbor set that appears in Proposition 2.3(1) and Theorem 2.4(1), i.e., in the six-neighbor cases.

An easy way to visualize these results is by considering the following pictures, whose exact definitions will be given in Sect. 5. Depict a vertex for each isometry of $\Gamma$ and draw an edge between two vertices if the corresponding tiles intersect each other. We obtain the pictures of Figs. 1, 2, 3 anf 4 (see Propositions 4.1 and 4.4 for the choice of the vertices in the six-neighbor cases). Disk-likeness happens iff $\mathcal{D}$ is connected along the full edges; the remaining edges are dashed.

The assumptions of Proposition 2.3 were shown in [4] to be minimal. It is also the case for our Theorem 2.4. As noticed in Remark 2.5.1, we listed all possible disklike cases of $p 2$-tiles. We illustrate the seven-neighbor case in Fig. 5. Each tile $T$ is the union of nine squares of side size 1/3 (gray on the figure). Using the expansion $g(x, y)=(6 x, 6 y)$, one sees that $g(T)$ is the union of 36 isometric copies of $T$. The example on the left of Fig. 5 is disk-like: $T$ has seven neighbors with the shape of Theorem 2.4(2), and the digit set, which has 36 elements, is connected along the full edges of Fig. 4. Modifying a bit the digit set, one gets Gelbrich's 36- $p 2$-reptile. The tile still has the seven neighbors indicated in Theorem 2.4(2), but the digit set is no more connected along the full edges of the picture.

The paper is now organized as follows. In Sect. 3 we give a proof of Theorem 2.1: six is the least number of neighbors of a lattice or a $p 2$-tile. In Sect. 4 we show that the shape of the neighbor set $\mathcal{S}$ of a plane lattice tile is fixed by the least number of neighbors and that this is also the case for a $p 2$-tile if it is supposed to be connected. 
Fig. 1 Proposition 2.3

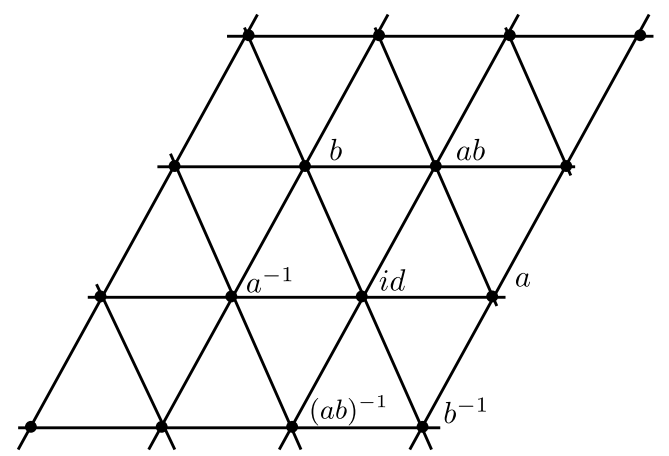

(1) Six-neighbor case (see also Proposition 4.1).

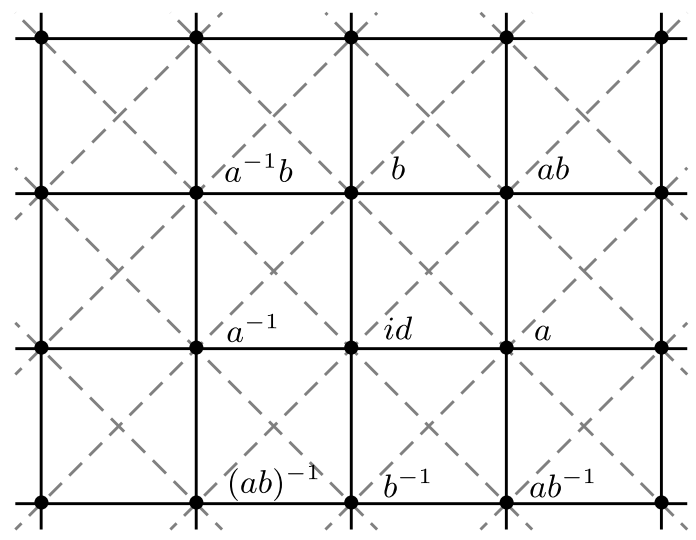

(2) Eight-neighbor case.

Fig. 2 Theorem 2.4(1), six-neighbor case; see also Proposition 4.4

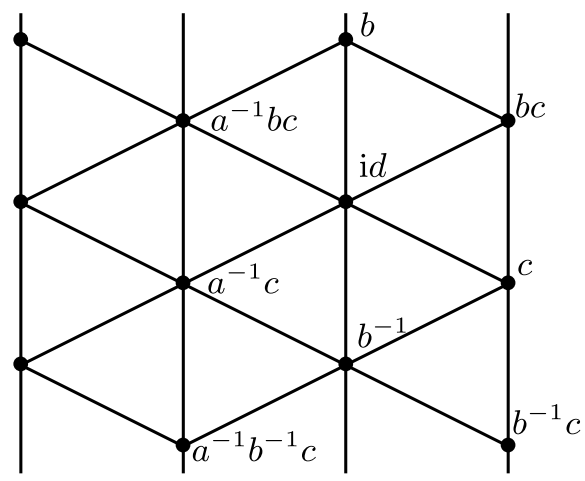

In Sect. 5 we define graphs and drawings associated to a crystile and the generated tiling. We use these graphs in Sect. 6, where we give a new proof of Bandt and Wang's result and the proof of its extension to $p 2$-crystiles. Section 7 presents some examples and ends up with comments and new questions. 


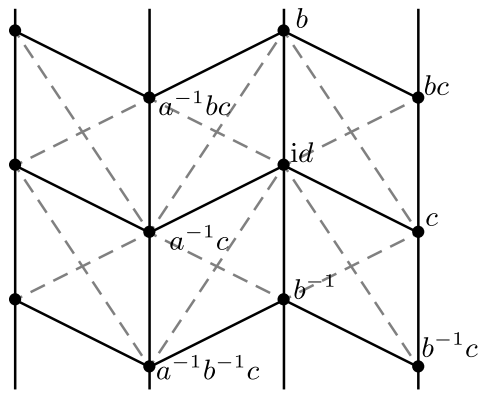

Shape 1.

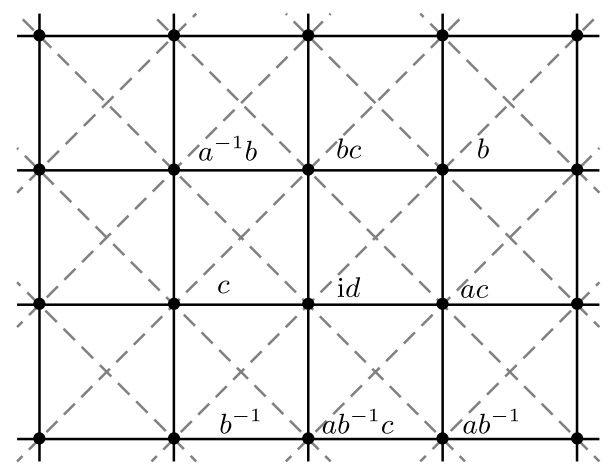

Shape 2.

Fig. 3 Theorem 2.4(3), 8-neighbor case

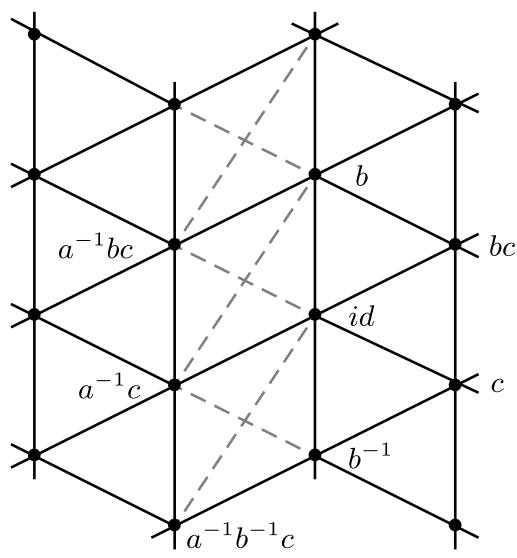

(2) 7 neighbor case.

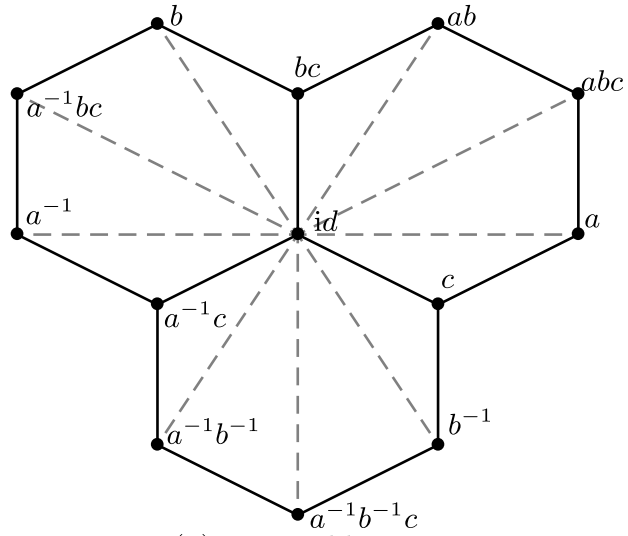

(4) 12 neighbor case.

Fig. 4 Theorem 2.4((2), (4))
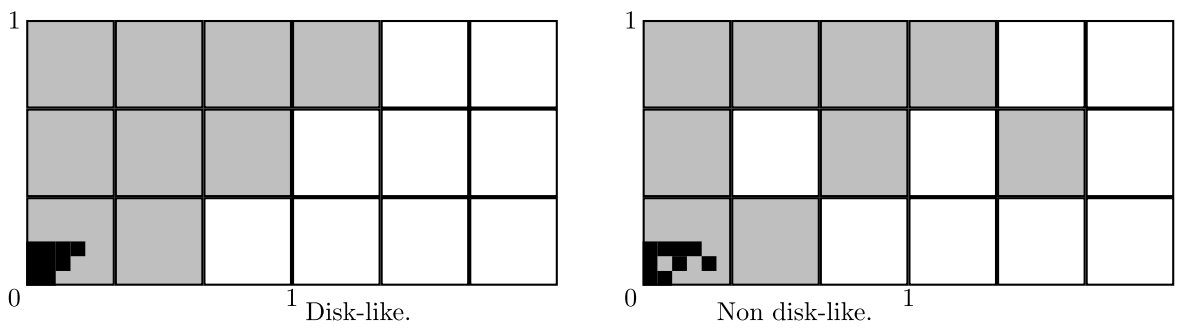

Fig. 5 36- $p 2$-reptiles with seven neighbors 


\section{Least Neighbor Number}

We are interested in the least number of neighbors a tile has in a lattice or a $p 2$-tiling. We will show that for tilings using a single compact tile, this number is six. Let $\{\gamma(T): \gamma \in \Gamma\}$ be a tiling of $\mathbb{R}^{n}$ which uses a single compact prototile $T$. For $d \geq 1$, the set $V_{d}$ of $d+1$-folded points is the set of points belonging to $T$ and to $d$ distinct other tiles $\gamma_{i}(T)(1 \leq i \leq d)$. Note that $V_{1}$ is in fact the boundary of the tile $T$. $V_{2}$ is the set of double, $V_{3}$ of triple points. Sets of vertices have been recently studied, for example, in [6], where the Hausdorff dimension of $V_{d}$ was computed.

It is clear that a tiling of $\mathbb{R}$ by compact pieces has double points. Lebesgue's covering theorem [7, Theorem 1.8.15, p. 78] says that if $\mathcal{C}$ is a finite closed cover of the $n$-cube $[0,1]^{n}$ no member of which meets two opposite faces of $[0,1]^{n}$, then $\mathcal{C}$ contains $n+1$ elements with a nonempty intersection. See also [1] for a recent improvement of this result. As a corollary of this, we can infer the following proposition.

Proposition 3.1 For each tiling $\{\gamma(T): \gamma \in \Gamma\}$ of $\mathbb{R}^{n}$ which uses a single compact prototile $T$, the set of $(n+1)$-folded points is nonempty.

Proposition 3.2 Let $T$ be a compact tile providing a tiling of the plane by a lattice $\Gamma$. Then its set of neighbors $\mathcal{S}$ has at least six elements.

Proof Suppose that $\mathcal{S}$ has less than six elements. Then $\mathcal{S}=\left\{a, a^{-1}, b, b^{-1}\right\}$ for two independent translations $a, b \in \Gamma$ because $\Gamma=\langle\mathcal{S}\rangle$. By the existence of triple points, two intersection sets $T \cap a^{i}(T)$ and $T \cap b^{j}(T)$ (or $T \cap a^{k}(T)$ ) must intersect for some $i, j \in\{-1,1\}$ (or distinct $i, k \in\{-1,1\}$ ); however, this leads to the existence of a new neighbor $a^{i} b^{-j}(T)$ (or $\left.a^{i-k}(T)\right)$.

We now consider the case of $p 2$-tiles.

Proposition 3.3 Let $T$ be a compact tile providing a tiling of the plane by a p2-group $\Gamma$. Then the set of neighbors $\mathcal{S}$ has at least six elements.

Proof Assume that $\Gamma$ is generated by the two translations $u, v$ and the rotation $r$ of Definition 1.1. Then, one can easily check the commutation rules $u^{i} v^{j}=v^{j} u^{i}$ and $u^{i} v^{j} r=r u^{-i} v^{-j}$ for all $i, j \in \mathbb{Z}$. Moreover, if both $\gamma$ and $\gamma^{\prime}$ are $\pi$-rotations, $\gamma^{-1} \gamma^{\prime}$ is a translation, and if exactly one of them is a translation, then $\gamma^{-1} \gamma^{\prime}$ is a $\pi$-rotation. We will also often use the fact that from $\gamma(T) \cap \gamma^{\prime}(T) \neq \emptyset$ it follows that $\gamma^{-1} \gamma^{\prime} \in \mathcal{S}$.

Since the neighbor set $\mathcal{S}$ of $T$ generates $\Gamma$, it contains an element $u^{i_{0}} v^{j_{0}} r=\eta r$, where $\eta=u^{i_{0}} v^{j_{0}}$ is a translation. Let $T^{\prime}=T \cup \eta r(T)$. Then $\left\{u^{i} v^{j}\left(T^{\prime}\right): i, j \in \mathbb{Z}\right\}$ is a lattice tiling of the plane.

Obviously, if $T^{\prime}$ has ten neighbors or more, then $T$ has at least six neighbors.

If $T^{\prime}$ has eight neighbors, say $\left\{u^{i_{k}} v^{j_{k}}\left(T^{\prime}\right), 1 \leq k \leq 8\right\}=\left\{\gamma_{k}^{ \pm 1}\left(T^{\prime}\right), 1 \leq k \leq 4\right\}$, then $T \cup \eta r(T)$ intersects $\gamma_{k}(T) \cup \gamma_{k} \eta r(T)$ for all $k \in\{1, \ldots, 4\}$, and thus for each $k$, either $\left\{\gamma_{k}^{ \pm 1}\right\} \subset \mathcal{S}$, or $\gamma_{k}^{-1} \eta r \in \mathcal{S}$, or $\gamma_{k} \eta r \in \mathcal{S}$. If $\gamma_{k} \in \mathcal{S}$ for some $k \in\{1,2,3,4\}$, then $\mathcal{S}$ has at least six elements. If $\gamma_{k} \notin \mathcal{S}$ for each $k \in\{1,2,3,4\}$, we have $\gamma_{k}^{i_{k}} \eta r \in \mathcal{S}$ for $i_{1}, i_{2}, i_{3}, i_{4} \in\{1,-1\}$. Then $\mathcal{S}$ has a sixth element. Otherwise, $\# \mathcal{S}=5$, and by 
the existence of triple points, $T \cap \gamma_{j}^{i_{j}} \eta r(T)$ must intersect $T \cap \gamma_{k}^{i_{k}} \eta r(T)$ for some $j, k \in\{1,2,3,4\}$ and $i_{j} \in\{-1,0,1\}, i_{k} \in\{-1,1\}$. This indicates that $\mathcal{S}$ has a sixth element $\gamma_{j}^{i_{j}} \gamma_{k}^{-i_{k}}$

If $T^{\prime}$ has exactly six neighbors, there exist $\alpha, \beta \in \Gamma=\langle u, v\rangle$ such that $\alpha^{ \pm 1}\left(T^{\prime}\right)$, $\beta^{ \pm 1}\left(T^{\prime}\right), \delta^{ \pm 1}\left(T^{\prime}\right)=(\alpha \beta)^{ \pm 1}\left(T^{\prime}\right)$ are the six neighbors of $T^{\prime}$ (see Proposition 4.1 of this paper). Let $\mathcal{F}_{1}=\left\{\alpha^{ \pm 1}, \beta^{ \pm 1}, \delta^{ \pm 1}\right\}$. Then, $T$ has at least four neighbors

$$
\eta r(T), \quad \alpha^{i_{1}}(\eta r)^{k_{1}}(T), \quad \beta^{i_{2}}(\eta r)^{k_{2}}(T), \quad \delta^{i_{3}}(\eta r)^{k_{3}}(T)
$$

for some $k_{1}, k_{2}, k_{3} \in\{0,1\}$ and $i_{1}, i_{2}, i_{3} \in\{-1,1\}$.

We claim that there is at least one translation $\gamma \in \mathcal{S}$. Otherwise, the existence of triple points indicates that $T \cap \operatorname{tr}(T)$ intersects $T \cap t^{\prime} r(T)$ for some $t c \neq t^{\prime} c \in \mathcal{S}$, where $t, t^{\prime}$ are translations. Then, $\operatorname{tr}(T) \cap t^{\prime} r(T) \neq \emptyset$ and $t^{-1} t^{\prime}(T) \cap T \neq \emptyset$, and thus $t^{-1} t^{\prime} \in \mathcal{S}$.

By this claim, if $k_{j} \neq 0$ for each $j \in\{1,2,3\}, \mathcal{S}$ has a translation $\gamma$ and thus has at least the following six elements:

$$
\eta r, \quad \gamma^{ \pm 1}, \quad \alpha^{i_{1}}(\eta r)^{k_{1}}, \quad \beta^{i_{2}}(\eta r)^{k_{2}}, \quad \delta^{i_{3}}(\eta r)^{k_{3}} .
$$

If two of $k_{1}, k_{2}, k_{3}$ are 0 , say $k_{1}=k_{2}=0$, then $\mathcal{S}$ has at least the six elements

$$
\eta r, \quad \alpha^{ \pm 1}, \quad \beta^{ \pm 1}, \quad \delta^{i_{3}}(\eta r)^{k_{3}} .
$$

If now exactly one of $k_{1}, k_{2}, k_{3}$ is 0 , say $k_{1}=0$, then $\mathcal{S}$ has at least the five elements

$$
\eta r, \quad \alpha^{ \pm 1}, \quad \beta^{i_{2}}(\eta r), \quad \delta^{i_{3}}(\eta r)
$$

where $\delta=\alpha \beta$. If $\mathcal{S}$ has a sixth element, we are done. We suppose on the contrary that

$$
\mathcal{S}=\left\{\eta r, \alpha^{ \pm 1}, \beta^{i_{2}}(\eta r), \delta^{i_{3}}(\eta r)\right\} .
$$

Consider $T^{\prime \prime}=T \cup \beta^{i_{2}} \eta r(T)$, which tiles $\mathbb{R}^{2}$ under the action of the lattice $\langle\alpha, \beta\rangle$. Then, $\beta^{i_{2}} \eta r(T)$ has exactly five neighbors

$$
\beta^{i_{2}}(T), \quad \alpha^{ \pm 1} \beta^{i_{2}} \eta r(T), \quad T, \quad \beta^{i_{2}} \delta^{-i_{3}}(T),
$$

and hence $T^{\prime \prime}$ has exactly six neighbors

$$
\beta^{ \pm 1}\left(T^{\prime \prime}\right), \quad \alpha^{ \pm 1}\left(T^{\prime \prime}\right), \quad\left(\delta^{i_{3}} \beta^{-i_{2}}\right)^{ \pm 1}\left(T^{\prime \prime}\right) .
$$

We can infer from Proposition 4.1 of the next section that $\delta^{i_{3}} \beta^{-i_{2}} \in\left\{(\alpha \beta)^{ \pm 1}\right.$, $\left.\left(\alpha \beta^{-1}\right)^{ \pm 1}\right\}$. This is impossible, since $i_{2}, i_{3} \in\{-1,1\}$.

\section{Neighbor Set}

In this section, we show that the shape of the neighbor set of a compact lattice or compact connected $p 2$-tile is already fixed by the fact that it contains the least number of elements (i.e., six elements). 
Proposition 4.1 Let $T$ be a compact tile providing a tiling of the plane by a lattice. If the set $\mathcal{S}$ of neighbors of $T$ has exactly six elements, then

$$
\mathcal{S}=\left\{a, a^{-1}, b, b^{-1}, a b,(a b)^{ \pm 1}\right\}
$$

for some $a, b \in \Gamma$ with $\Gamma=\langle a, b\rangle$.

Proof By Proposition 3.2 of the preceding section, we already know that there exist two elements $a, b \in \mathcal{S} \subset \Gamma$ such that $\Gamma=\langle a, b\rangle$ and $\mathcal{S}=\left\{a^{ \pm 1}, b^{ \pm 1}\right\} \cup\left\{\delta^{ \pm 1}\right\}$ for some $\delta \in \Gamma$. We just need to certify that $\delta$ has the form $(a b)^{ \pm 1}$ or $\left(a b^{-1}\right)^{ \pm 1}$.

First note that $T \cap a(T)$ does not intersect $T \cap a^{-1}(T)$. Otherwise, $\delta=a^{2}$, and the union $Q$ of all those compact sets $a^{k}(T \cap b(T))$ with $k \in \mathbb{Z}$ is a closed set such that $\mathbb{R}^{2} \backslash Q$ has two unbounded components. Let

$$
Q_{0}=\bigcup_{k \leq 0} a^{k}(T \cap b(T)), \quad Q_{+}=\bigcup_{k>0} a^{k}(T \cap b(T)) .
$$

Then, one can see that both $\mathbb{R}^{2} \backslash Q_{0}$ and $\mathbb{R}^{2} \backslash Q_{+}$have exactly one unbounded component. That is to say, there are two points in the sphere separated by $\bar{Q}=Q \cup\{\infty\}=$ $\overline{Q_{0}} \cup \overline{Q_{+}}$which cannot be separated by $\overline{Q_{0}}=Q_{0} \cup\{\infty\}$ or $\overline{Q_{+}}=Q_{+} \cup\{\infty\}$ alone. Since the sphere is a Janizewski space (see [13, Sect. 61]), this implies that $Q_{0}=\bigcup_{k \leq 0} a^{k}(T \cap b(T))$ and $Q_{+}=\bigcup_{k>0} a^{k}(T \cap b(T))$ must intersect each other.

Therefore, $a^{i} b(T) \cap a^{j}(T) \neq \varnothing$ for some $i \leq 0$ and $j>0$, and thus $a^{j-i} b \in \mathcal{S}$ with $j-i \geq 1$, which would increase the number of neighbor. Similarly, $T \cap b(T)$ does not intersect $T \cap b^{-1}(T)$.

If now $T \cap a^{i}(T)$ and $T \cap b^{j}(T)$ intersect for $i, j \in\{-1,1\}$, then $a b \in \mathcal{S}$ or $a b^{-1} \in \mathcal{S}$, and thus $\delta$ or $\delta^{-1}$ is equal to $a b$ or $a b^{-1}$.

Suppose that $\left[T \cap a^{i}(T)\right] \cap\left[T \cap b^{j}(T)\right]=\emptyset$ for every $i, j \in\{-1,1\}$. Then by the existence of triple points, $T \cap a^{i}(T)$ or $T \cap b^{j}(T)$ must intersect $[T \cap \delta(T)] \cup$ $\left[T \cap \delta^{-1}(T)\right]$. We may assume without loss of generality that $T \cap a(T)$ intersects $T \cap \delta(T)$. Then, $a(T) \cap \delta(T) \neq \emptyset$ and $a^{-1} \delta \in \mathcal{S}$, while $\delta \notin\left\{a^{ \pm 1}, b^{ \pm 1}\right\}$. Thus we have $a^{-1} \delta \in\left\{b, b^{-1}\right\}$, that is to say, $\delta=a b$ or $\delta=a b^{-1}$ again.

In the case of $p 2$-tiles, we require the tiles to be connected. Indeed, we will use the following lemma.

Lemma 4.2 Let $T$ be a connected compact set and $\{\gamma(T): \gamma \in \Gamma\}$ be a tiling of $\mathbb{R}^{n}$ for some countable group of isometries $\Gamma$. Then the boundary $\partial T$ of $T$ is connected.

Proof This follows from the unicoherence of Euclidean spaces (see [16]). Recall that a connected space is unicoherent if, representing it as any union of two closed connected sets, the intersection of these sets is connected. Thus, writing $\mathbb{R}^{n}=T \cup$ $\bigcup_{\gamma \neq \mathrm{id}} \gamma(T)$, we obtain that $\partial T=T \cap \bigcup_{\gamma \neq \mathrm{id}} \gamma(T)$ is connected.

Let us consider a tiling of the plane by a single tile $T$ and a group of isometries $\Gamma$. We denote by $E_{\gamma}$ the compact intersection set $T \cap \gamma(T)$ for $\gamma \in \mathcal{S}$ and by $\partial T$ the boundary of $T$. 
Lemma 4.3 Suppose that the neighbor set of $T$ contains $m \geq 2$ elements $\gamma_{1}, \ldots, \gamma_{m}$ and that $\partial T$ is connected. Then each $E_{\gamma_{i}}, i=1, \ldots, m$, intersects at least one $E_{\gamma_{j}}, j \neq i$.

Proof Writing for the boundary $\partial T=\bigcup_{i=1}^{m} E_{\gamma_{i}}$, this follows from the connectivity of $\partial T$.

Now $\Gamma$ is supposed to be a $p 2$-group.

Proposition 4.4 If $T$ is a connected compact set which is the central tile of a p2-tiling and if the neighbor set $\mathcal{S}$ of $T$ contains exactly six elements, then $\mathcal{S}$ has the following shape, where $a, b$ are independent translations and $c$ is $a \pi$-rotation:

$$
\mathcal{S}=\left\{b^{ \pm 1}, c, a^{-1} c, b c, a^{-1} b c\right\} .
$$

Proof At least one translation $b \neq 1$ and a $\pi$-rotation $c$ must belong to the neighbor set. Indeed, if $\mathcal{S}$ contains only translations, it cannot generate a $p 2$-group. If it contains only rotations, then by the existence of triple points, two neighbors $\gamma(T), \gamma^{\prime}(T)$ of $T$ must intersect, and thus the translation $\gamma^{-1} \gamma^{\prime}$ should also be a neighbor. Since if $\gamma(T)$ intersects $T$, then $\gamma^{-1}(T)$ also intersects $T$, we obtain that $\left\{b, b^{-1}, c\right\} \subset \mathcal{S}$.

We now show that no other translation can be in $\mathcal{S}$. To this matter, we suppose the contrary, i.e., that

$$
\mathcal{S}=\left\{b, b^{-1}, a, a^{-1}, c, c^{\prime}\right\},
$$

where $a \neq \mathrm{id}, b, b^{-1}$ are translations, and $c^{\prime}$ a $\pi$-rotation. Two cases may occur.

If $a$ and $b$ are dependent, then the neighbor set contains four translations that are linearly dependent and two rotations. Using Lemma 4.3, one rotation can be written in terms of the translation $b$ and of the other rotation. This contradicts the fact that $\mathcal{S}$ is a generating set for $\Gamma$.

If $a$ and $b$ are independent, then the set $E_{b}$ cannot intersect the sets $E_{b^{-1}}, E_{a}, E_{a^{-1}}$. Therefore, it intersects $E_{c}$ or $E_{c^{\prime}}$, and thus $c^{\prime}=b^{-1} c$ or $c=b^{-1} c^{\prime}$. Doing the same with the set $E_{a}$, we obtain that also $c^{\prime}=a^{-1} c$ or $c=a^{-1} c^{\prime}$. This is a contradiction, since $a \neq b, b^{-1}$.

Consequently, we can write for the neighbor set:

$$
\mathcal{S}=\left\{b, b^{-1}, c, c_{1}, c_{2}, c_{3}\right\},
$$

where $c_{1}, c_{2}, c_{3}$ are the remaining $\pi$-rotations. If the set $E_{c}$ intersects $E_{b}$ or $E_{b^{-1}}$, then one of the remaining rotations must be $b^{ \pm 1} c$; if it intersects one of the sets $E_{\gamma}$ associated to the remaining rotations, say $\gamma=c_{1}$, then $c c_{1}=b^{ \pm 1}$, so $c_{1}=b^{ \pm 1} c$. Since by Lemma 4.3 one of these possibilities occurs, we can suppose w.l.o.g. (by exchanging $b$ and $b^{-1}$ ) that $b c$ belongs to the neighbor set, and thus

$$
\mathcal{S}=\left\{b, b^{-1}, c, b c, c_{2}, c_{3}\right\}
$$

To obtain a $\pi$-rotation that can be written in terms of a translation $a$ independent of $b$, let us consider the tile $T^{\prime}=T \cup c(T)$. This tile provides a lattice tiling of the 
plane: there are independent translations $a^{\prime}, b^{\prime} \neq$ id such that

$$
\mathbb{R}^{2}=\bigcup_{(i, j) \in \mathbb{Z}^{2}} a^{i} b^{\prime j}\left(T^{\prime}\right)
$$

By Remark 1.3 for a lattice tiling, there must be a neighbor of the central tile that is not a translation by some powers of $b$, i.e., there is $a \neq$ id independent of $b$ such that $a\left(T^{\prime}\right) \cap T^{\prime} \neq \emptyset$. This leads, as $a \notin \mathcal{S}$, to $a c \in \mathcal{S}$ or $a^{-1} c \in \mathcal{S}$. W.1.o.g. (exchange $a$ and $a^{-1}$ ), we suppose that $a^{-1} c \in \mathcal{S}$ is such that now

$$
\mathcal{S}=\left\{b, b^{-1}, c, b c, a^{-1} c, c_{3}\right\}
$$

The rotation $c_{3}$ can be written with the help of $a, b$, and $c$. Indeed, the set $E_{a^{-1} c}$ cannot intersect the sets $E_{c}$ and $E_{b c}$ (this would introduce new translations in the set of neighbors), thus it must have nonempty intersection with $E_{b}, E_{b^{-1}}$, or $E_{c_{3}}$. In all these cases we obtain that $c_{3}=a^{-1} b^{ \pm 1} c$, and thus $\mathcal{S}$ has one of the shapes of our proposition (replace $a$ by $a b$ if $c_{3}=a^{-1} b^{-1} c$ ).

\section{Definition of Graphs}

We now define graphs associated to the tilings and their corresponding drawings. As noticed in Sect. 2, these allow us to visualize the configuration of the tiles in a given tiling. They will constitute the key of our proof of Proposition 2.3 and Theorem 2.4 in the next section.

A graph is a pair $(V, E)$ of sets such that $E \subset V \times V$. The elements $x$ of $V$ are called vertices, these of $E$ are called edges and written $x y$ or $y x$. Two vertices are incident if they belong to a same edge. If $V^{\prime} \subset V$ and $E^{\prime}$ is the set containing all the edges $x y \in E$ with $x, y \in V^{\prime}$, then the graph $G^{\prime}=\left(V^{\prime}, E^{\prime}\right)$ is called the induced subgraph of $G$ by $V^{\prime}$.

For a crystallographic tiling $\{\gamma(T): \gamma \in \Gamma\}$ which uses a single prototile $T$, the set $\mathcal{S}$ of neighbors of the central tile and the set $\mathcal{A}$ of its adjacent neighbors are symmetric sets, i.e., $\mathcal{S}=\mathcal{S}^{-1}$ and $\mathcal{A}=\mathcal{A}^{-1}$. This leads to the definition of the following graphs.

Definition 5.1 For a finite symmetric set $M \subset \Gamma$, we define $G(M)$ as the graph with vertex set $\Gamma$ and for which a 2-element set $\left\{\gamma_{1}, \gamma_{2}\right\} \subset \Gamma$ is an edge whenever $\gamma_{1}^{-1} \gamma_{2} \in M$. For the particular choice $M=\mathcal{S}, G_{N}:=G(\mathcal{S})$ is the neighbor graph, and for $M=\mathcal{A}, G_{A}:=G(\mathcal{A})$ is the adjacent graph of the tiling. If $\mathcal{A}^{\prime} \subset \mathcal{A}$, we will write $G_{A}\left(\mathcal{A}^{\prime}\right):=G\left(\mathcal{A}^{\prime}\right)$ for the corresponding subgraph of $G_{A}$. Note that $G_{N}$ and $G_{A}$ are regular graphs by the group property of $\Gamma$ : each vertex is incident with the same number of edges, called the degree of the graph.

Remark 5.2 The neighbor graph $G_{N}$ is always connected; otherwise, choosing a component of $G_{N}$ with vertex set $\mathcal{V}_{0}, \bigcup_{\gamma \in \mathcal{V}_{0}} \gamma(T)$ would be a subset of $\mathbb{R}^{n}$ which is both closed and open, contradicting the connectivity of $n$-dimensional Euclidean spaces. 
Definition 5.3 Let $G=(V, E)$ be a graph with set of vertices $V$ and set of edges $E$. If there is a mapping $\pi:(V, E) \rightarrow \mathbb{R}^{2}$ such that $\pi(V)$ is a discrete set of the plane, $\pi(x y)$ is a simple arc joining $\pi(x)$ and $\pi(y)$, and

$$
\pi(x y) \cap \pi(u v)=\{\pi(x), \pi(y)\} \cap\{\pi(u), \pi(v)\} \quad(x y, u v \in E \text { disjoint }),
$$

then we say that $G$ is planar and call $\pi(G)$ (or shortly $\pi$ ) a drawing of $G$. For every planar graph $G$ with a drawing $\pi$, the set $\mathbb{R}^{2} \backslash \pi(G)$ is an open set; its components are the faces of $\pi(G)$ (or $G$ ).

Definition 5.4 Given a planar graph $G=(V, E)$ and a drawing $\pi$ of $G$, we consider the derived graph of $G$ defined as the graph $G_{1}=\left(V, E_{1}\right)$ emerging from $G$ with the same set of vertices and where two vertices $x, y$ are incident if their images $\pi(x), \pi(y)$ belong to the closure of the same face of $G . E_{1}$ contains $E$, and we extend $\pi$ to a map $\pi_{1}$ on $E_{1}$ by joining the images of vertices corresponding to a new edge by a simple open arc inside one of their common faces. This extension gives rise to a picture of the graph $G_{1}$.

\section{Proof of Bandt-Wang-Like Statements}

This section is devoted to the proof of Proposition 2.3 and Theorem 2.4 stated in Sect. 2. We first recall a result of [4] and [14], where adjacent neighbors of lattice and $p 2$-tiles having a neighbor set of known shape could be identified. Then, we give a formulation of the new criterion proved in [15] under weaker assumptions. The proof eventually follows.

If the shape of the neighbor set of the central tile is given, it is possible to identify all or some of the adjacent neighbors of this tile, as stated in the following propositions.

Proposition 6.1 [4, Lemma 3.3] Let $T$ be a connected tile providing a lattice tiling. Let $a, b$ be two independent translations:

(i) If $T$ has six neighbors

$$
\mathcal{S}=\left\{a^{ \pm 1}, b^{ \pm 1},\left(a b^{-1}\right)^{ \pm 1}\right\}
$$

then $\mathcal{S}$ consists of adjacent neighbors of id.

(ii) If $T$ has eight neighbors

$$
\mathcal{S}=\left\{a^{ \pm 1}, b^{ \pm 1},\left(a b^{-1}\right)^{ \pm 1},\left(a^{-1} b\right)^{ \pm 1}\right\}
$$

then $\left\{a^{ \pm 1}, b^{ \pm 1}\right\}$ are adjacent neighbors of id.

This proposition was proved in [4] by an $\epsilon$-argument. We mention that a shorter proof can be given using directly the connectedness of the boundary of $\partial T$ as in [14, Proposition 5.4]. The connectedness of $\partial T$ is indeed assured by Lemma 4.2 and the connectedness of $T$. 
Proposition 6.2 [14, Proposition 5.4] Let $T$ be a connected p2-tile. Let $a, b$ be two independent translations and $c$ a $\pi$-rotation.

(i) If T has six neighbors

$$
\mathcal{S}=\left\{b^{ \pm 1}, c, a^{-1} c, b c, a^{-1} b c\right\}
$$

then $\mathcal{S}$ consists of adjacent neighbors of id.

(ii) If $T$ has seven neighbors $\mathcal{S}=\left\{b^{ \pm 1}, c, b c, a^{-1} c, a^{-1} b c, a^{-1} b^{-1} c\right\}$, then $\left\{b^{ \pm 1}, c, b c, a^{-1} c\right\}$ are adjacent neighbors of id.

(iii) If $T$ has eight neighbors $\mathcal{S}=\left\{b^{ \pm 1}, c, a^{-1} c, b c, b^{-1} c, a^{-1} b c, a^{-1} b^{-1} c\right\}$ (resp. $\left.\mathcal{S}=\left\{c, b c, a c, a b^{-1} c, b^{ \pm 1},\left(a^{-1} b\right)^{ \pm 1}\right\}\right)$

then $\left\{b^{ \pm 1}, c, a^{-1} c\right\}$ (resp. $\left.\left\{c, b c, a c, a b^{-1} c\right\}\right)$ are adjacent neighbors of id.

(iv) If $T$ has twelve neighbors $\left\{c, a^{-1} c, b c, a b c, a^{-1} b c, a^{-1} b^{-1} c, a^{ \pm 1}, b^{ \pm 1}\right.$, $\left.(a b)^{ \pm 1}\right\}$, then $\left\{c, a^{-1} c, b c\right\}$ are adjacent neighbors of id.

The criterion of disk-likeness as it is stated in [15] involves the knowledge of all adjacent neighbors of the central tile. Since Proposition 6.2 only identifies some of them, we cannot apply this criterion directly. Before giving a formulation of this criterion with modified assumptions, we recall some definitions and notation that can be also found in [15].

Definition 6.3 Let $G_{A}^{\prime}=(\Gamma, E)$ be a subgraph of the adjacent graph $G_{A}$ of a crystallographic tiling. Suppose that $G_{A}^{\prime}$ has a drawing $\pi$ for which the following holds: there is $p \in \mathbb{R}^{2}$ with $\gamma_{1}(p) \neq \gamma_{2}(p)$ for all $\gamma_{1}, \gamma_{2} \in \Gamma, \gamma_{1} \neq \gamma_{2}$, such that:

- $\pi(\gamma)=\gamma(p) \quad(\gamma \in \Gamma)$;

- there is a constant $c \in \mathbb{R}$ such that for all $e \in E$ joining the vertices $x$ and $y$, we have

$$
\pi(e) \subset B_{c}(x) \cap B_{c}(y),
$$

where $B_{r}(x):=\left\{y \in \mathbb{R}^{2}:|y-x| \leq r\right\}$ denotes a closed disk with radius $r$ centered at $x$.

Then $\pi$ is called an admissible drawing of $G_{A}^{\prime}$. Moreover, we also call an extension $\pi_{1}$ of $\pi$ admissible if it satisfies (6.1) for all $e \in E_{1}, E_{1}$ being the set of edges of the derived graph of $G_{A}^{\prime}$ (see Definition 5.4).

The following criterion is derived from the criterion in [15, Theorem 2.4].

Proposition 6.4 Assume that $T$ is a plane crystallographic reptile with respect to a crystallographic group $\Gamma$, an expanding affine map $g$, and a digit set $\mathcal{D}$. We write $\mathcal{D}^{k}$ for the set of isometries such that

$$
g^{k}(T)=\bigcup_{\delta \in \mathcal{D}^{k}} \delta(T) .
$$

Let $\mathcal{A}$ denote the set of adjacent neighbors of the central tile. Then $T$ is disk-like if and only if there is a subset $\mathcal{A}^{\prime}$ of $\mathcal{A}$ with $\mathcal{A}^{\prime-1}=\mathcal{A}^{\prime}$ such that the following three conditions hold: 
(i) The subgraph $G_{A}\left(\mathcal{A}^{\prime}\right)$ of the adjacent graph is a connected planar graph.

(ii) For every $k \in \mathbb{N}$, the set $\mathcal{D}^{k}$ induces a connected subgraph in $G_{A}\left(\mathcal{A}^{\prime}\right)$.

(iii) $G_{A}\left(\mathcal{A}^{\prime}\right)$ has an admissible draw $\pi: G_{A}\left(\mathcal{A}^{\prime}\right) \rightarrow \mathbb{R}^{2}$ such that the derived graph of $G_{A}\left(\mathcal{A}^{\prime}\right)$ is exactly the neighbor graph $G_{N}$.

Remark 6.5 Condition (iii) says that two tiles $\gamma_{1}(T), \gamma_{2}(T)$ are neighbors if and only if the vertices $\pi\left(\gamma_{1}\right), \pi\left(\gamma_{2}\right)$ lie on the boundary of a single face of the drawing $\pi\left(G_{A}\left(\mathcal{A}^{\prime}\right)\right)$. Note that the necessity part is simply proved by taking $\mathcal{A}^{\prime}=\mathcal{A}$ and applying [15, Theorem 2.4].

We apply this proposition in order to reprove Proposition 2.3 and to prove its extension to the $p 2$-crystiles, Theorem 2.4. As we noticed in Remark 2.5 , if $T$ is disk-like, the sets $\mathcal{A}$ are given in Grünbaum and Shephard's classification. The main difficulty lies in the proof of item (ii), i.e., the $\mathcal{A}^{\prime}$-connectivity of the iterates $\mathcal{D}^{k}$ when $\mathcal{D}$ is assumed to be $\mathcal{A}^{\prime}$-connected. This part is the purpose of the following two lemmata.

We use the notation of Proposition 6.4.

Lemma 6.6 Suppose that the collections $\mathcal{D} \cup \mathcal{D}_{\gamma}$ are $\mathcal{A}^{\prime}$-connected for every $\gamma \in \mathcal{A}^{\prime}$. Then so also is $\mathcal{D}^{k}$ for all $k \in \mathbb{N}$ (see Remark 1.3 for the definition of $\mathcal{D}_{\gamma}$ ).

Proof We prove that $\mathcal{D}^{2}$ is $\mathcal{A}^{\prime}$-connected if $\mathcal{D}=\mathcal{D}^{1}$ is. The result then follows by induction on $k$ : writing $\mathcal{D}^{k+1}=\bigcup_{\gamma \in \mathcal{D}^{k}} \mathcal{D}_{\gamma}$, one shows similarly that $\mathcal{D}^{k+1}$ is $\mathcal{A}^{\prime}$-connected if $\mathcal{D}^{k}$ is.

First note that if a set $M \subset \Gamma$ is $\mathcal{A}^{\prime}$-connected, then so is $\gamma M$ for all $\gamma \in \Gamma$. Let $d_{1}, d_{2} \in \Gamma$ with $d_{1}^{-1} d_{2} \in \mathcal{A}^{\prime}$. Taking $M=\mathcal{D} \cup \mathcal{D}_{d_{1}^{-1} d_{2}}$ and $\gamma=g d_{1} g^{-1}$, we see that $\mathcal{D}_{d_{1}} \cup \mathcal{D}_{d_{2}}$ is $\mathcal{A}^{\prime}$-connected. Write now that

$$
\mathcal{D}^{2}=\bigcup_{\gamma \in \mathcal{D}^{1}} \mathcal{D}_{\gamma}
$$

and choose $\gamma \in \mathcal{D}^{1}$ with $\gamma \neq \mathrm{id}$. By the $\mathcal{A}^{\prime}$-connectedness of $\mathcal{D}^{1}$, there is a chain of elements $\gamma_{1}, \ldots, \gamma_{n}$ in $\mathcal{D}^{1}$ from $\gamma_{1}=$ id to $\gamma_{n}=\gamma$ such that $\gamma_{i}^{-1} \gamma_{i+1} \in \mathcal{A}^{\prime}$. Thus $\mathcal{D} \cup \cdots \cup \mathcal{D}_{\gamma} \subset \mathcal{D}^{2}$ is $\mathcal{A}^{\prime}$-connected. Doing this for each $\gamma \in \mathcal{D}^{1} \backslash\{\mathrm{id}\}$, we obtain that $\mathcal{D}^{2}$ is $\mathcal{A}^{\prime}$-connected.

Lemma 6.7 Suppose that the crystile $T$ has the neighbor set $\mathcal{S}$ and that $\mathcal{D}$ is $\mathcal{A}^{\prime}$-connected, where the pairs $\left(\mathcal{S}, \mathcal{A}^{\prime}\right)$ are read off from the items Proposition 2.3(2) and Theorem 2.4(2-4). Then the collections $\mathcal{D} \cup \mathcal{D}_{\gamma}$ are $\mathcal{A}^{\prime}$-connected for every $\gamma \in \mathcal{A}^{\prime}$.

Proof We first state some properties valid for all the considered pairs $\left(\mathcal{S}, \mathcal{A}^{\prime}\right)$ and then give separate proofs.

First note that since $\mathcal{D}$ is a complete set of coset representatives of $\Gamma / g \Gamma g^{-1}$, we have

$$
\begin{aligned}
& \mathcal{D}_{\gamma} \cap \mathcal{D}_{\gamma}^{\prime}=\emptyset \quad \text { for } \gamma \neq \gamma^{\prime}, \\
& \forall \gamma \in \Gamma, \exists ! \gamma^{\prime} \in \Gamma \quad \text { such that } \gamma \in \mathcal{D}_{\gamma^{\prime}} .
\end{aligned}
$$


For each constellation $\left(\mathcal{S}, \mathcal{A}^{\prime}\right)$, we consider a drawing $\pi$ associated to $G_{A}\left(\mathcal{A}^{\prime}\right)$. In the sense of Definition 5.4 and by assumption on the neighbor set $\mathcal{S}$, a picture of the neighbor graph $G_{N}$ is obtained by adding all the diagonal line segments in each of the non-gasket-like simple loops, as depicted in Figs. 1, 4, and 3 (in the figures, we write $\gamma$ for $\pi(\gamma)$ ). This picture then corresponds to an extension $\pi_{1}$ of $\pi$ and was already represented in Figs. 1, 2, 3 and 4. Clearly, the degree of $G_{N}$ is then $s:=\# \mathcal{S}$. Moreover, for each connected subgraph of $G_{N}$ with vertex set $\mathcal{D}^{\prime}$, finite or infinite, the collection of vertices $\bigcup_{d \in \mathcal{D}^{\prime}} \mathcal{D}_{d}$ induces a connected subgraph in $G_{N}$. We will use this fact at the end of the proof. Remember that "induces" means that we connect the vertices of $\mathcal{D}$ via all the available edges in $G_{N}$ (so even if $\mathcal{D}$ is $\mathcal{A}^{\prime}$-connected, crossing diagonals may appear in the picture of the subgraph of $G_{N}$ induced by $\mathcal{D}$ ).

One can easily see that this picture has the following properties:

(i) Two lines $\pi_{1}\left(x_{1} x_{2}\right)$ and $\pi_{1}\left(y_{1} y_{2}\right)$ intersect if and only if they have a common vertex or they are the two diagonal line segments in the same face of $\pi\left(G_{A}\right)$.

(ii) $G_{N}$ is $s$-connected in the sense that for any $\mathcal{U} \subset \Gamma$ which has less than $s$ elements, the subgraph induced by $\Gamma \backslash \mathcal{U}$ is connected.

(iii) Suppose that $L=\left(V_{1}, E_{1}\right)$ is an arbitrary connected subgraph of $G_{A}\left(\mathcal{A}^{\prime}\right)$ with finite vertex set $V_{1}$. If each edge $x y \in E_{1}$ satisfies $x^{-1} y \in \mathcal{A}^{\prime}$ and if $\mathbb{R}^{2} \backslash \pi(L)$ has a bounded component intersecting $\pi(G)$ containing a point $\pi(\gamma)$ for some $\gamma \in G$, then $G \backslash V_{1}$ induces a disconnected subgraph in $G_{N}$ which has a component of finite vertex set containing $\gamma$. We can see that the image $\pi(L)$ of $L$ must contain a loop, so we will in the sequel refer to this as Loop Property.

We now distinguish the constellations.

Lattice-8 Neighbor-Case Let us consider first the pair $\left(\mathcal{S}, \mathcal{A}^{\prime}\right)$ of Proposition 2.3(2) and suppose that $\mathcal{D} \cup \mathcal{D}_{a}$ is not $\left\{a^{ \pm 1}, b^{ \pm 1}\right\}$-connected. Since $g(T)=\bigcup_{d \in \mathcal{D}} d(T)$ and $g a(T)=\bigcup_{d^{\prime} \in \mathcal{D}_{a}} d^{\prime}(T)$ intersect each other, we can choose some $\delta \in \mathcal{D}$ and $\delta^{\prime} \in \mathcal{D}_{a}$ with $\delta(T) \cap \delta^{\prime}(T) \neq \emptyset$. Then, we must have $\delta^{-1} \delta^{\prime} \in\left\{(a b)^{ \pm 1},\left(a b^{-1}\right)^{ \pm 1}\right\}=\mathcal{S} \backslash \mathcal{A}^{\prime}$. We may assume that $\delta=\mathrm{id}, \delta^{\prime}=a b$. See Fig. 1 for relative positions of id and $a b$.

Let $\alpha, \beta \in \Gamma$ be the uniquely determined elements with $a \in \mathcal{D}_{\alpha}, b \in \mathcal{D}_{\beta}$. Because $\mathcal{D} \cup \mathcal{D}_{a}$ is not $\left\{a^{ \pm 1}, b^{ \pm 1}\right\}$-connected, $\alpha$ and $\beta$ are both distinct from id and $a$.

Then, we can find a vertex $c \in \Gamma \backslash\{\mathrm{id}, a, \alpha, \beta\}$ such that $c(T) \cap T \neq \emptyset$ and $c(T) \cap$ $a(T) \neq \emptyset$. We can see on Fig. 1 that $c$ must belong to $\left\{b, a b, b^{-1}, a b^{-1}\right\}$.

Now, we can choose

$$
d \in \mathcal{D}, \quad d_{1}, d_{2} \in \mathcal{D}_{c}, \quad d_{3} \in \mathcal{D}_{a}
$$

such that

$$
d(T) \cap d_{1}(T) \neq \emptyset, \quad d_{2}(T) \cap d_{3}(T) \neq \emptyset .
$$

Then, $\left\{d^{-1} d_{1}, d_{2}^{-1} d_{3}\right\} \subset\left\{a^{ \pm 1}, b^{ \pm 1},(a b)^{ \pm 1},\left(a b^{-1}\right)^{ \pm 1}\right\}$. By the $\left\{a^{ \pm 1}, b^{ \pm 1}\right\}$-connectivity of $\mathcal{D}_{\gamma}$ for every $\gamma \in \Gamma$, consider the three connected pieces $H_{\mathrm{id}}, H_{a}, H_{c}$ in the picture of $G_{N}$ joining all the vertices $\mathcal{D}, \mathcal{D}_{a}, \mathcal{D}_{c}$, respectively, and whose lines $\pi(x y)$ are defined for vertices $x, y \in \mathcal{D}$ (or $\mathcal{D}_{a}$, or $\mathcal{D}_{c}$ ) whenever $x y^{-1} \in\left\{a^{ \pm 1}, b^{ \pm 1}\right\}$. We can find three disjoint simple paths $P \subset H_{\mathrm{id}}, P_{a} \subset H_{a}$, and $P_{c} \subset H_{c}$ such that: 
- the two end points of $P$ are $\pi(d)$ and $\pi(\mathrm{id})$;

- the two end points of $P_{c}$ are $\pi\left(d_{1}\right)$ and $\pi\left(d_{2}\right)$;

- the two end points of $P_{a}$ are $\pi\left(d_{3}\right)$ and $\pi(a b)$.

Note that $\pi(a)$ and $\pi(b)$ do not lie on these paths.

Therefore, the union $J$ of $P \cup P_{a} \cup P_{c}$ with the three line segments $\pi_{1}\left(d d_{1}\right)$, $\pi_{1}\left(d_{2} d_{3}\right), \pi_{1}(\operatorname{id}(a b))$ is a simple closed curve. Since the diagonal line segments $\pi_{1}(a b)$ and $\pi_{1}(\operatorname{id}(a b))$ intersect each other at a single point, the two points $\pi(a), \pi(b)$ are separated by $J$.

Since $\pi(a)$ and $\pi(b)$ (or equivalently, $\pi\left(\mathcal{D}_{\alpha}\right)$ and $\pi\left(\mathcal{D}_{\beta}\right)$ ) are separated by the loop $J$, we may assume that $\pi(a)$ is in the interior of $J$, and thus the picture of the subgraph induced by $\mathcal{D}_{\alpha}$ is enclosed in the interior of $J$, according to the picture of $G_{N}$.

Note that $\pi_{1}\left(d d_{1}\right), \pi_{1}\left(d_{2} d_{3}\right)$ could be either diagonal or vertical or horizontal line segments (see Fig. 6).

We discuss the possible three cases separately as follows.

Case 1 Suppose that $\pi_{1}\left(d d_{1}\right), \pi_{1}\left(d_{2} d_{3}\right)$ are both vertical or horizontal, i.e., $\mathcal{D} \cup \mathcal{D}_{c}$ and $\mathcal{D}_{c} \cup \mathcal{D}_{a}$ are $\mathcal{A}^{\prime}$-connected. Then the union $J_{1}$ of $P \cup P_{a} \cup P_{c}$ with the vertical or horizontal line segments $\pi_{1}\left(d d_{1}\right), \pi_{1}\left(d_{2} d_{3}\right), \pi_{1}(b(\mathrm{id})), \pi_{1}(b(a b))$ satisfies the condition in "Loop Property." Then, we can claim that $\Gamma \backslash\left(\mathcal{D} \cup \mathcal{D}_{a} \cup \mathcal{D}_{c} \cup \mathcal{D}_{\beta}\right)$ induces a disconnected subgraph in $G_{N}$. Otherwise, there would be a simple path $P$ disjoint from all the $\pi_{1}$-images of the vertices of $\mathcal{V}=\mathcal{D} \cup \mathcal{D}_{a} \cup \mathcal{D}_{c} \cup \mathcal{D}_{\beta}$ which connects the vertex $\pi_{1}(a)$ to a vertex $\pi_{1}(\eta)$ in the exterior of $J$. We may assume without loss of generality that all the vertices $\pi_{1}(u)$ on the path $P$ other than $\pi_{1}(\eta)$ belong to the interior of $J$. Then, $\gamma(T) \cap \eta(T) \neq \emptyset$ for some $\pi_{1}(\gamma) \neq \pi_{1}(\eta)$ on the path $P$, where $\pi_{1}(\gamma)$ lies in the interior of $J$. This is a contradiction to "Loop Property."

Case 2 Suppose that one of $\pi_{1}\left(d d_{1}\right), \pi_{1}\left(d_{2} d_{3}\right)$ is diagonal and the other is vertical or horizontal, say

$$
d d_{1}^{-1} \in\left\{(a b)^{ \pm 1},\left(a b^{-1}\right)^{ \pm 1}\right\} .
$$

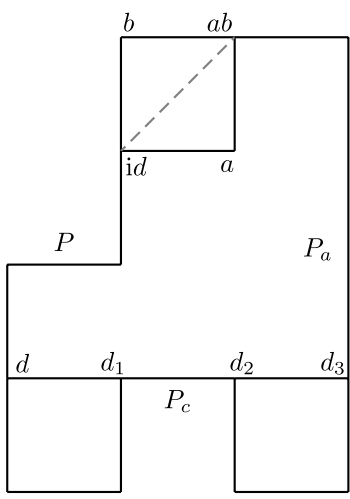

Case 1.
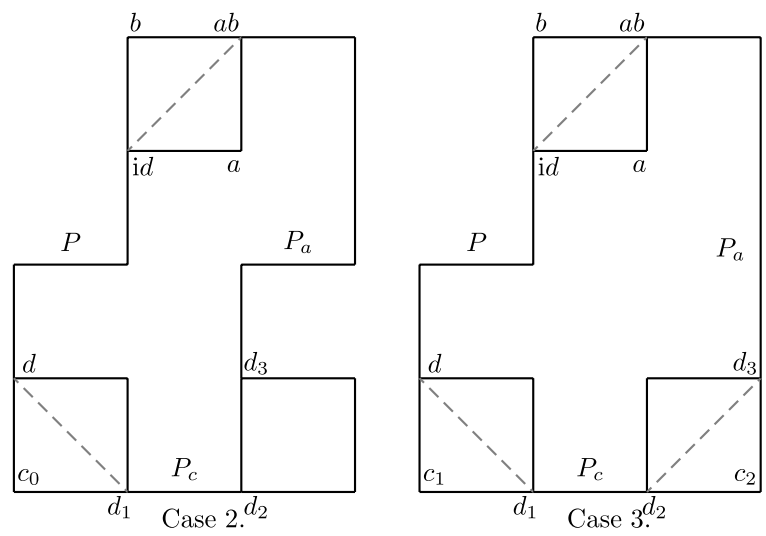

Fig. 6 Lemma 6.7, lattice-8 neighbor-case 
This means $\mathcal{D} \cup \mathcal{D}_{c}$ is not $\mathcal{A}^{\prime}$-connected, but $\mathcal{D}_{c} \cup \mathcal{D}_{a}$ is. We can choose $\pi_{1}\left(c_{0}\right)$ on $J$ or in the exterior of $J$ such that $d^{-1} c_{0}, c_{0}^{-1} d_{1} \in\left\{a^{ \pm 1}, b^{ \pm 1}\right\}$. Then, the union $J_{2}$ of $P \cup P_{a} \cup P_{c}$ with the vertical or horizontal line segments $\pi_{1}\left(d c_{0}\right), \pi_{1}\left(c_{0} d_{1}\right)$, $\pi_{1}\left(d_{2} d_{3}\right), \pi_{1}(b(\mathrm{id})), \pi_{1}(b(a b))$ satisfies the condition in "Loop Property." By the argument used in Case 1, we can infer that

$$
\Gamma \backslash\left(\mathcal{D} \cup \mathcal{D}_{\gamma} \cup \mathcal{D}_{a} \cup \mathcal{D}_{c} \cup \mathcal{D}_{\beta}\right)
$$

induces a disconnected subgraph in $G_{N}$, where $\gamma \in \Gamma$ is the unique element with $c_{0} \in \mathcal{D}_{\gamma}$.

Case 3 Suppose that $\pi_{1}\left(d d_{1}\right), \pi_{1}\left(d_{2} d_{3}\right)$ are both diagonal line segments, i.e., $\mathcal{D} \cup \mathcal{D}_{c}$ and $\mathcal{D}_{c} \cup \mathcal{D}_{a}$ are not $\mathcal{A}^{\prime}$-connected. Then we can find $\pi_{1}\left(c_{1}\right), \pi_{1}\left(c_{2}\right)$ in the exterior of $J$ according to the drawing of $G_{N}$ such that

$$
\left\{d^{-1} c_{1}, c_{1}^{-1} d_{1}\right\} \subset\left\{a^{ \pm 1}, b^{ \pm 1}\right\}, \quad\left\{d_{2}^{-1} c_{2}, c_{2}^{-1} d_{3}\right\} \subset\left\{a^{ \pm 1}, b^{ \pm 1}\right\} .
$$

Then, the union $J_{3}$ of $P \cup P_{a} \cup P_{c}$ with the vertical or horizontal line segments

$$
\pi_{1}\left(d c_{1}\right), \quad \pi_{1}\left(c_{1} d_{1}\right), \quad \pi_{1}\left(d_{2} c_{2}\right), \quad \pi_{1}\left(c_{2} d_{3}\right), \quad \pi_{1}(b(\mathrm{id})), \quad \pi_{1}(b(a b))
$$

satisfies the condition in "Loop Property." Let $\gamma_{1}, \gamma_{2}$ be the two uniquely determined elements of $\Gamma$ with

$$
c_{1} \in \mathcal{D}_{\gamma_{1}}, \quad c_{2} \in \mathcal{D}_{\gamma_{2}} .
$$

Put

$$
\mathcal{V}:=\mathcal{D} \cup \mathcal{D}_{a} \cup \mathcal{D}_{c} \cup \mathcal{D}_{\beta} \cup \mathcal{D}_{\gamma_{1}} \cup \mathcal{D}_{\gamma_{2}}
$$

Then $\Gamma \backslash \mathcal{V}$ induces a disconnected subgraph in $G_{N}$. By the argument used in Case 1, we can infer that $\Gamma \backslash\left(\mathcal{D} \cup \mathcal{D}_{a} \cup \mathcal{D}_{c} \cup \mathcal{D}_{\beta}\right)$ induces a disconnected subgraph in $G_{N}$.

In each of the above three cases, we always have some $\mathcal{V}$, the union of $\mathcal{D}_{\gamma}$ with $\gamma$ running through a collection of at most six elements of $\Gamma$ such that the infinite collection $\Gamma \backslash \mathcal{V}$ induces a disconnected subgraph in $G_{N}$. This contradicts the eightconnectivity of $G_{N}$, as mentioned at the beginning of the proof.

Hence $\mathcal{D} \cup \mathcal{D}_{a}$ is $\left\{a^{ \pm 1}, b^{ \pm 1}\right\}$-connected. The cases of $\mathcal{D} \cup \mathcal{D}_{a^{-1}}, \mathcal{D} \cup \mathcal{D}_{b}, \mathcal{D} \cup \mathcal{D}_{b^{-1}}$ are treated similarly.

p2-7 Neighbor-Case Secondly we deal with the constellation $\left(\mathcal{S}, \mathcal{A}^{\prime}\right)$ of Theorem 2.4(2). We want to show that $\mathcal{D} \cup \mathcal{D}_{\gamma}$ induces a connected subgraph in $G_{A}\left(\mathcal{A}^{\prime}\right)$ for each $\gamma \in \mathcal{A}^{\prime}=\left\{b, b^{-1}, c, b c, a^{-1} c\right\}$ (see Fig. 4).

If $\gamma=b^{ \pm 1}$ or $\gamma=a^{-1} c$, this can be obtained in a similar way as for the preceding lattice eight-neighbor case; this is due to the fact that id and $\gamma$ have "enough" common neighbors for these values of $\gamma$, namely three. Let $\gamma=c$, which has only two common neighbors with id (the same kind of argument holds for $\gamma=b c$ ), and suppose that $\mathcal{D} \cup \mathcal{D}_{c}$ is not $\mathcal{A}^{\prime}$-connected. Since $T \cap c(T) \neq \varnothing$, we have $g(T) \cap$ $g c(T) \neq \emptyset$, and thus there exist $d \in \mathcal{D}$ and $d^{\prime} \in \mathcal{D}_{c}$ such that $d(T) \cap d^{\prime}(T) \neq \emptyset$. In our assumption, $d^{-1} d^{\prime} \in \mathcal{S} \backslash \mathcal{A}^{\prime}$. Without loss of generality. either $\left\{d, d_{1}\right\}=\left\{\mathrm{id}, a^{-1} b c\right\}$ or $\left\{d, d_{1}\right\}=\left\{\mathrm{id}, a^{-1} b^{-1} c\right\}$. 
We assume that $d=$ id and $d_{1}=a^{-1} b c$ (the treatment of $d_{1}=a^{-1} b^{-1} c$ runs likewise). Let $\eta \in \Gamma$ be the uniquely determined element with $b \in \mathcal{D}_{\eta}$. Then $\eta \notin\{$ id, $c\}$, otherwise $\mathcal{D} \cup \mathcal{D}_{c}$ would be $\mathcal{A}^{\prime}$-connected in $G_{A}$. Since $b \in \mathcal{D}_{\eta}$, both intersections $g \eta(T) \cap g(T)$ and $g \eta(T) \cap g c(T)$ are nonempty, thus taking their images by $g^{-1}$, we see that $\eta$ must be a common neighbor of id and $c$, i.e., $\eta \in\left\{b c, b^{-1}\right\}$. Similarly, let $\eta^{\prime}$ with $a^{-1} c \in \mathcal{D}_{\eta}$; then $\eta^{\prime} \in\left\{b c, b^{-1}\right\}$.

We claim that $\eta=\eta^{\prime}$. Indeed, if $\eta \neq \eta^{\prime}$, by the assumption on $\mathcal{S}$ the intersection $\eta(T) \cap \eta^{\prime}(T)$ must be empty, thus so has to be its blow-up by $g$. But this is not the case, because $b \in \mathcal{D}_{\eta}, a^{-1} c \in \mathcal{D}_{\eta^{\prime}}$, and $b(T) \cap a^{-1} c(T) \neq \emptyset$. Consequently, $\eta=\eta^{\prime}$.

$\mathcal{D}_{\eta}$ being $\mathcal{A}^{\prime}$-connected, there is a simple path $P$ in $G_{A}\left(\mathcal{A}^{\prime}\right)$ from $\pi(b)$ to $\pi\left(a^{-1} c\right)$ such that all vertices in $P$ belong to $\pi\left(\mathcal{D}_{\eta}\right)$. Since id, $a^{-1} b c \notin \mathcal{D}_{\eta}$, either $P^{\prime}$, the union of $P$ with the broken line from $\pi(b)$ to $\pi\left(a^{-1} c\right)$ via $\pi$ (id), encloses $\pi\left(a^{-1} b c\right)$, or $P^{\prime \prime}$, the union of $P$ with the broken line from $\pi(b)$ to $\pi\left(a^{-1} c\right)$ via $\pi\left(a^{-1} b c\right)$, encloses $\pi$ (id). Thus, by the "Loop Property," either $\Gamma \backslash\left(\mathcal{D}_{\eta} \cup \mathcal{D}\right)$ or $\Gamma \backslash\left(\mathcal{D}_{\eta} \cup \mathcal{D}_{c}\right)$ is disconnected in $G_{N}$. This contradicts the seven-connectivity of $G_{N}$. Hence $\mathcal{D} \cup \mathcal{D}_{c}$ is also $\mathcal{A}^{\prime}$-connected for every $\gamma \in \mathcal{A}^{\prime}$.

p2-8 Neighbor-Case The constellation of Theorem 2.4(3) is treated as in the lattice case (see Fig. 3).

p2-12 Neighbor-Case We eventually deal with the constellation $\left(S, \mathcal{A}^{\prime}\right)$ of Theorem 2.4(4).

Assume that $\mathcal{D} \cup \mathcal{D}_{\alpha}$ is not $\mathcal{A}^{\prime}$-connected for some $\alpha \in \mathcal{A}^{\prime}$. Since $\mathcal{D}$ is $\mathcal{S}$-connected, $\mathcal{D} \cup \mathcal{D}_{\alpha}$ must be $\mathcal{S}$-connected. Thus, there exist some $d_{1} \in \mathcal{D}$ and $a_{2} \in \mathcal{D}_{\alpha}$ with $d_{1}^{-1} a_{2} \in\left(\mathcal{S} \backslash \mathcal{A}^{\prime}\right)$. That is to say, in the drawing $\pi$ of $G_{A}\left(\mathcal{A}^{\prime}\right), \pi\left(d_{1}\right)$ and $\pi\left(a_{2}\right)$ lie on the boundary of the same face $F_{1}$ but not on a single side of $F_{1}$. See Fig. 7 for the three possible relative positions of $d_{1}$ and $a_{2}$. Moreover, If $d^{\prime} \in \mathcal{D}$ and $a^{\prime} \in \mathcal{D}_{\alpha}$ are neighbors, we must have $d^{\prime-1} a^{\prime} \in\left(\mathcal{S} \backslash \mathcal{A}^{\prime}\right)$, and thus $\pi\left(d^{\prime}\right)$ and $\pi\left(a^{\prime}\right)$ lie on boundary of the same face of $\pi\left(G_{A}\left(\mathcal{A}^{\prime}\right)\right)$ and not on a single side.

Clearly, $\partial F_{1} \backslash\left\{\pi\left(d_{1}\right), \pi\left(a_{2}\right)\right\}$ is the union of two open polygonal arcs, each of which contains at least one element of $\pi(G)$.

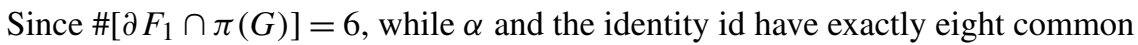
neighbors, we can choose a common neighbor $\beta$ of $\alpha$ and id with $\mathcal{D}_{\beta} \cap \pi^{-1}\left(F_{1}\right)=\emptyset$.

Now, we can choose $d_{2} \in \mathcal{D}, b_{1}, b_{2} \in \mathcal{D}_{\beta}$, and $a_{1} \in \mathcal{D}_{\alpha}$ with $\left\{b_{1}^{-1} d_{2}, a_{1}^{-1} b_{2}\right\} \subset \mathcal{S}$. Let $F_{2}, F_{3}$ be the faces of $\pi\left(G_{A}\left(\mathcal{A}^{\prime}\right)\right)$ containing $\left\{\pi\left(b_{1}\right), \pi\left(d_{2}\right)\right\}$ and $\left\{\pi\left(b_{2}\right), \pi\left(a_{1}\right)\right\}$, respectively. Since $\mathcal{D}_{\beta} \cap \pi^{-1}\left(F_{1}\right)=\emptyset, F_{1} \notin\left\{F_{2}, F_{3}\right\}$.

By the $\mathcal{A}^{\prime}$-connectedness of $\mathcal{D}$, we can find three simple paths $P, P_{\alpha}, P_{\beta}$ in drawings of the subgraphs of $G_{A}\left(\mathcal{A}^{\prime}\right)$ respectively generated by $\mathcal{D}, \mathcal{D}_{\alpha}, \mathcal{D}_{\beta}$ such that:

Fig. 7 Lemma 6.7, $p 2-12$ neighbor-case. Relative positions of $d_{1}$ and $a_{2}$ on $F_{1}$

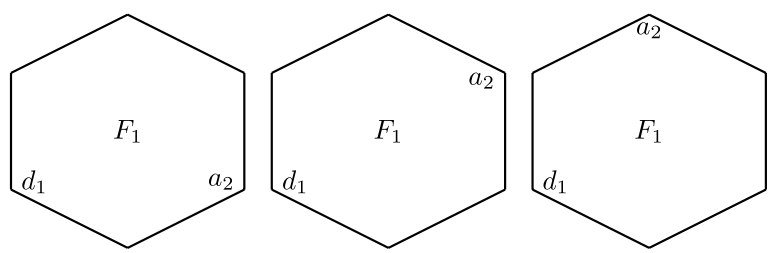


- $\pi\left(d_{1}\right), \pi\left(d_{2}\right)$ are the two ends of $P$

- $\pi\left(a_{1}\right), \pi\left(a_{2}\right)$ are the two ends of $P_{\alpha}$, and

- $\pi\left(b_{1}\right), \pi\left(b_{2}\right)$ are the two ends of $P_{\beta}$.

Note that the above three paths could be degenerate ones, like the case $d_{1}=d_{2}$, and thus $P=\left\{\pi\left(d_{1}\right)\right\}$.

Claim $1 F_{2} \neq F_{3}$. Otherwise, we would have $a_{1}^{-1} d_{2} \in\left(\mathcal{S} \backslash \mathcal{A}^{\prime}\right)$, and $\partial F_{2} \backslash\left\{a_{1}, d_{2}\right\}$ would consist of two open polygonal arcs each of which contains at least one element of $\pi(G) \cap \partial F_{2}$. (Similarly in the case of $F_{1}$.) Then, the union $J_{0}$ of $P \cup P_{\alpha}$ with the two line segments $\pi_{1}\left(a_{2} d_{1}\right) \subset F_{1}$ and $\pi_{1}\left(d_{2} a_{1}\right) \subset F_{2}$ is a polygonal simple closed curve in the plane whose interior $\operatorname{Int}\left(J_{0}\right)$ contains at least two elements, $\pi\left(\gamma_{1}\right) \in \pi(G) \cap F_{1}$ and $\pi\left(\gamma_{2}\right) \in \pi(G) \cap F_{2}$.

Proof Since $\left(F_{i} \backslash \overline{\operatorname{Int}\left(J_{0}\right)}\right) \cap \pi(G)$ has at most three elements for $i=1$, 2, we may denote by $e_{1}, e_{2}, \ldots, e_{k}(k \leq 6)$ the elements of $G$ with $\pi\left(e_{i}\right) \in\left(F_{1} \cup F_{2}\right) \backslash \overline{\operatorname{Int}\left(J_{0}\right)}$ and choose $\varepsilon_{i}(1 \leq i \leq k)$ of $G$ with $e_{i} \in \mathcal{D}_{\varepsilon_{i}}$.

For $i=1,2$, let $P_{i} \subset \partial F_{i}$ be an open subarc which is contained in $J_{0}$ 's exterior $\operatorname{Ext}\left(J_{0}\right)=\mathbb{R}^{2} \backslash \overline{\operatorname{Int}\left(J_{0}\right)}$. Then,

$$
P \cup P_{\alpha} \cup P_{1} \cup P_{2}
$$

satisfies the conditions of "Loop Property." This means that

$$
G \backslash\left(\{\text { id, } \alpha\} \cup\left\{\varepsilon_{1}, \varepsilon_{2}, \ldots, \varepsilon_{k}\right\}\right)
$$

induces a disconnected subgraph in $G_{N}$, a contradiction to the 12-connectivity of $G_{N}$. This proves Claim 1.

Now, let $J$ be the union of $P \cup P_{\alpha} \cup P_{\beta}$ with the line segments $\pi_{1}\left(a_{2} d_{1}\right), \pi_{1}\left(d_{2} b_{1}\right)$, and $\pi_{1}\left(b_{2} a_{1}\right)$. Then, $J$ is a polygonal simple closed curve in the plane with $\pi\left(\gamma_{1}\right) \in$ $\operatorname{Int}(J)$.

Claim $2\left\{b_{1}^{-1} d_{2}, a_{1}^{-1} b_{2}\right\} \subset\left(\mathcal{S} \backslash \mathcal{A}^{\prime}\right)$. Otherwise, say, $b_{1}^{-1} d_{2} \in \mathcal{A}^{\prime}$. Similar as for Claim 1 , there are $k \leq 6$ elements $e_{1}, e_{2}, \ldots, e_{k} \in G$ with $\pi\left(e_{i}\right)$ lying in $\left(F_{1} \cup F_{3} \backslash\right.$ $\overline{\operatorname{Int}(J)}$. We can see that

$$
G \backslash\left(\{\mathrm{id}, \alpha, \beta\} \cup\left\{\varepsilon_{1}, \varepsilon_{2}, \ldots, \varepsilon_{k}\right\}\right)
$$

induces a disconnected subgraph in $G_{N}$ which has a component with finite vertex set containing $\gamma_{1}$. This again contradicts the 12-connectivity of $G_{N}$.

Claim 2 immediately indicates that $\operatorname{Int}(J) \cap F_{i} \cap \pi(G)$ contains at least one element for $1 \leq i \leq 3$. Actually, we may further show that $\operatorname{Int}(J) \cap F_{i} \cap \pi(G)$ contains exactly one element for $1 \leq i \leq 3$. Otherwise, there would be $k \leq 8$ elements $e_{1}, e_{2}, \ldots, e_{k}$ with $\pi\left(e_{i}\right)$ lying in $\left(F_{1} \cup F_{2} \cup F_{3}\right) \backslash \overline{\operatorname{Int}(\mathrm{J})}$. If $\varepsilon_{i}(1 \leq i \leq k)$ are elements of $G$ with $e_{i} \in \mathcal{D}_{\varepsilon_{i}}$, where $\varepsilon_{i}=\varepsilon_{j}$ is possible, the subgraph of $G_{N}$ induced by

$$
G \backslash\left(\{\mathrm{id}, \alpha, \beta\} \cup\left\{\varepsilon_{1}, \varepsilon_{2}, \ldots, \varepsilon_{k}\right\}\right)
$$


Fig. 8 Lemma 6.7, $p 2-12$ neighbor-case

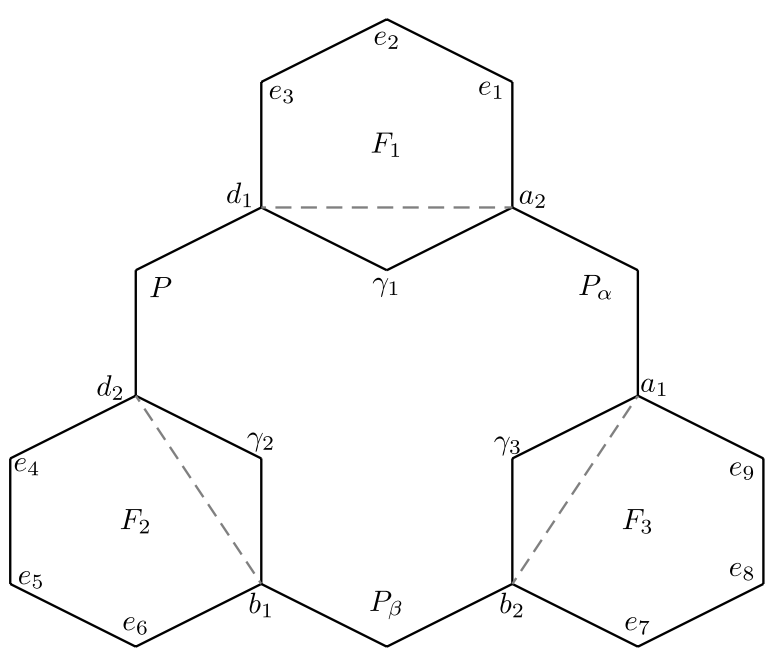

has a component with finite vertex set containing $\gamma_{1}$. This is impossible by the $12-$ connectivity of $G_{N}$.

Recall that $\left\{\pi\left(\gamma_{1}\right)\right\}=\operatorname{Int}(J) \cap F_{1} \cap \pi(G)$. We denote by $\gamma_{i}(i=2,3)$ the unique element of $G$ with $\pi\left(\gamma_{i}\right)$ lying in $\operatorname{Int}(J) \cap F_{i} \cap \pi(G)$. Here, $\gamma_{i}=\gamma_{j}$ is possible.

Let $e_{1}, e_{2}, \ldots, e_{9}$ be the nine elements of $G$ with $\pi\left(e_{i}\right)$ belonging to $\left(F_{1} \cup F_{2} \cup\right.$ $\left.F_{3}\right) \backslash \overline{\operatorname{Int}(J)}$, and $\varepsilon_{1}, \varepsilon_{2}, \ldots, \varepsilon_{9}$ the corresponding elements of $G$ with $e_{i} \in \mathcal{D}_{\varepsilon_{i}}$. Also, $\varepsilon_{i}=\varepsilon_{j}$ is possible here. Without losing generality, we may assume that

$$
\begin{aligned}
& \left\{\pi\left(e_{1}\right), \pi\left(e_{2}\right), \pi\left(e_{3}\right)\right\} \subset F_{1}, \quad\left\{\pi\left(e_{4}\right), \pi\left(e_{5}\right), \pi\left(e_{6}\right)\right\} \subset F_{2}, \\
& \left\{\pi\left(e_{7}\right), \pi\left(e_{8}\right), \pi\left(e_{9}\right)\right\} \subset F_{3} .
\end{aligned}
$$

See Fig. 8 for a rough depiction of the relative positions of $F_{1}, F_{2}, F_{3}$.

Claim $3\left\{\gamma_{1}, \gamma_{2}, \gamma_{3}\right\} \subset \mathcal{D}_{\delta}$ for a single $\delta \in \Gamma$. Otherwise, we may assume that $\gamma_{2} \in \mathcal{D}_{\delta^{\prime}}$ for some $\delta^{\prime} \neq \delta$. Then,

$$
G \backslash\left(\left\{\mathrm{id}, \alpha, \beta, \delta^{\prime}\right\} \cup\left\{\varepsilon_{1}, \varepsilon_{2}, \varepsilon_{3}, \varepsilon_{7}, \varepsilon_{8}, \varepsilon_{9}\right\}\right)
$$

would induce a disconnected subgraph in $G_{N}$, contradicting its 12-connectivity.

Claim $4 \varepsilon_{i} \neq \varepsilon_{j}$ for $i \neq j$. Otherwise, say, $\varepsilon_{1}=\varepsilon_{2}$; then $G \backslash\left\{\mathrm{id}, \alpha, \beta, \varepsilon_{2}, \varepsilon_{3}, \ldots, \varepsilon_{9}\right\}$ would induce a disconnected subgraph $G_{N}$, again impossible by 12-connectivity of $G_{N}$.

Claim $5\left\{\delta, \alpha^{-1} \delta, \beta^{-1} \delta\right\}=\mathcal{A}^{\prime}$. Otherwise, say, $\alpha^{-1} \delta \notin \mathcal{A}^{\prime}$; then, $\alpha$ and $\delta$ would not belong to a single edge of $G_{A}\left(\mathcal{A}^{\prime}\right)$, and thus $\alpha$ and $\delta$ would have exactly four common neighbors, see the preceding figure. This contradicts Claims 3 and 4 , which implies that $\alpha$ and $\delta$ have eight distinct common neighbors id, $\beta, \varepsilon_{1}, \varepsilon_{2}, \varepsilon_{3}, \varepsilon_{7}, \varepsilon_{8}, \varepsilon_{9}$. 
Conclusion Claim 5 is impossible. Indeed, $\alpha \in \mathcal{A}^{\prime}$ by the beginning assumption, and $\delta \in \mathcal{A}^{\prime}$ by Claim 5. However, $\mathcal{A}^{\prime}=\left\{c, a^{-1} c, b c\right\}$, and hence $\alpha$ and $\delta$ are $\pi$-rotations. Thus $\alpha^{-1} \delta$ is a translation and cannot belong to $\mathcal{A}^{\prime}$, a contradiction to Claim 5. This ends the proof of Lemma 6.7.

We are now ready to prove Proposition 2.3 and Theorem 2.4, using Proposition 6.4.

Proof of Proposition 2.3 and Theorem 2.4 We note that if $T$ is disk-like or if $\mathcal{D}$ is $\mathcal{F}$-connected (for some $\mathcal{F} \subset \mathcal{S}$ and hence for $\mathcal{F}=\mathcal{S}$ ), then $T$ is itself connected. Thus we can assume in this proof that the tile $T$ is connected.

(1) 6 Neighbor-Case Assume that $T$ has exactly six neighbors. In the lattice case, by Propositions 4.1 and 6.1 , the neighbor set is $\mathcal{S}=\left\{b, b^{-1}, a, a^{-1}, a^{-1} b, a b^{-1}\right\}$ for independent translations $a, b$ and only consists of adjacent neighbors: $\mathcal{S}=\mathcal{A}$. The graph $G_{A}$ has a drawing like in Fig. 1. The equivalence now follows from Proposition 6.4 by taking $\mathcal{A}^{\prime}=\mathcal{A}$ (in this case, condition (ii) of Proposition 6.4 reduces to $\left(\mathrm{ii}^{\prime}\right): \mathcal{D}$ is $\mathcal{S}$-connected). One can proceed similarly for the $p 2$-case (the drawing is given in Fig. 2).

(2) Other Cases In the lattice eight-neighbor case, assume that $T$ has exactly the neighbor set $\mathcal{S}=\left\{a^{ \pm 1}, b^{ \pm 1},(a b)^{ \pm 1},\left(b^{-1} a\right)^{ \pm 1}\right\}$. By Proposition $6.1, \mathcal{S}$ contains $\mathcal{A}^{\prime}:=\left\{a^{ \pm 1}, b^{ \pm 1}\right\}$ as adjacent neighbors. The corresponding graph $G_{A}\left(\mathcal{A}^{\prime}\right)$ has the drawing depicted in Fig. 1, where the dashed lines should be omitted.

If $T$ is disk-like, we have $\mathcal{A}^{\prime}=\mathcal{A}$, and the result follows from condition (ii) of Proposition $6.4\left(\mathcal{D}^{1}=\mathcal{D}\right)$. On the other side, suppose that $\mathcal{D}$ is $\mathcal{A}^{\prime}$-connected. Then the drawing of $G_{A}\left(\mathcal{A}^{\prime}\right)$ satisfies conditions (i) and (iii) of Proposition 6.4, as can be checked on Fig. 1. Condition (ii) is the purpose of Lemmata 6.6 and 6.7. By Proposition 6.4, $T$ is disk-like. The $p 2$-cases of 7, 8, and 12 neighbors can be treated in the same way.

\section{Examples and Comments}

We provide examples of disk-like and non-disk-like $p 2$-crystiles, before stating some comments and questions that arise from our study. In this section, the maps $u, v, r$ are defined by $u(x, y)=(x+1, y), v(x, y)=(x, y+1)$ and $r(x, y)=(-x,-y)$, as in Definition 1.1.

\subsection{Examples of Disk-Like $p 2$-Crystiles}

We are first interested in examples of disk-like $p 2$-crystiles.

6-Neighbor Case We consider the p2-crystile defined by the expansion map

$$
g(x, y)=(x-2 y+1 / 2, x+2 y-1 / 2)
$$



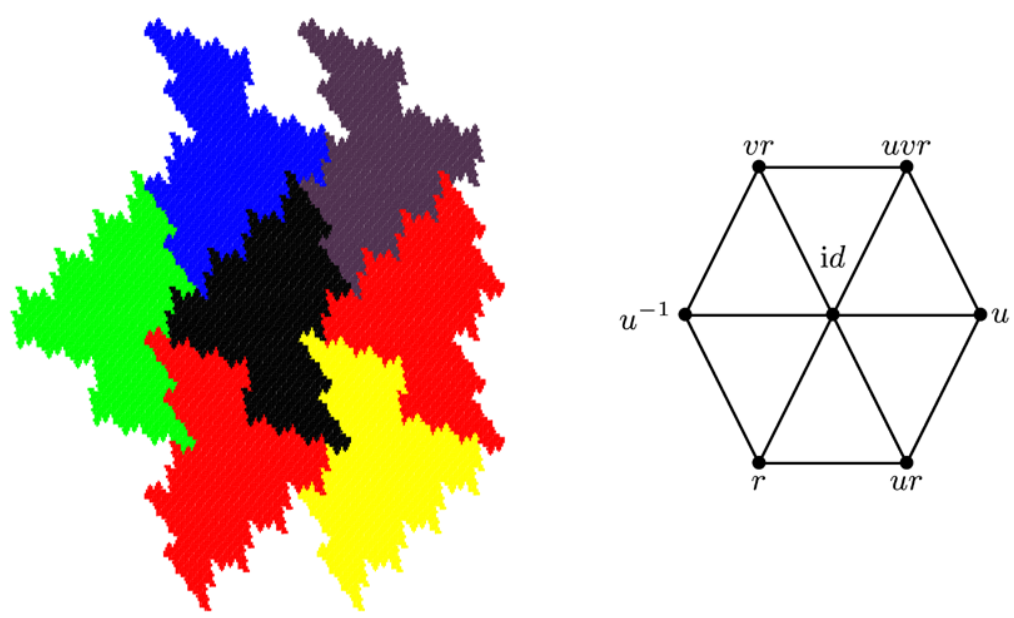

Fig. 9 Disk-like $p$ 2-crystile with six neighbors

and the digit set $\mathcal{D}=\{\mathrm{id}, r, u r, v r\}$. The crystile $T$ is solution of the equation

$$
g(T)=T \cup r(T) \cup u r(T) \cup v r(T) .
$$

With the methods developed in [14], we compute that the set of neighbors is

$$
\mathcal{S}=\left\{u^{ \pm 1}, r, u r, v r, u v r\right\}
$$

It has the shape given in Proposition 4.4 (take $a=v^{-1}, b=u, c=r$ ). The tile $T$, together with its neighbors, is depicted in Fig. 9. The digit set is $\mathcal{S}$-connected, as can be checked on the graph of the same figure. By Theorem 2.4(1), $T$ is disk-like.

Seven-Neighbor Case We consider an example that Gelbrich [8] listed as "not convincing," since he could not decide from the figure if it is disk-like or not. The expansion $g$ reads

$$
g(x, y)=\left(-x+y-\frac{1}{2},-2 x-y\right),
$$

and the digit set is $\mathcal{D}=\{\mathrm{id}, v, r\}$. The crystile $T$ and its neighbors are depicted in Fig. 10. By Theorem 2.4 (identify $u, v, r$ with $a, b, c$, respectively), $T$ is disk-like.

Eight-Neighbor Case A disk-like p2-crystile with eight neighbors of the first shape is obtained by considering the union of squares given on the left side of Fig. 11. The expansion map reads $g(x, y)=(4 x, 4 y)$, and the sixteen digits are easily read off from the picture.

Consider now the expansion $g(x, y)=(2 x+1 / 2,-x-2 y-1 / 2)$ and the digit set $\mathcal{D}=\{\mathrm{id}, r, u r, v r\}$. The corresponding crystile $T$ together with its neighbors is depicted in Fig. 12. The neighbor set has the second shape given in Theorem 2.4(3) (take $a=u v, b=v, c=r$ ), and $T$ is disk-like by Theorem 2.4(3). 

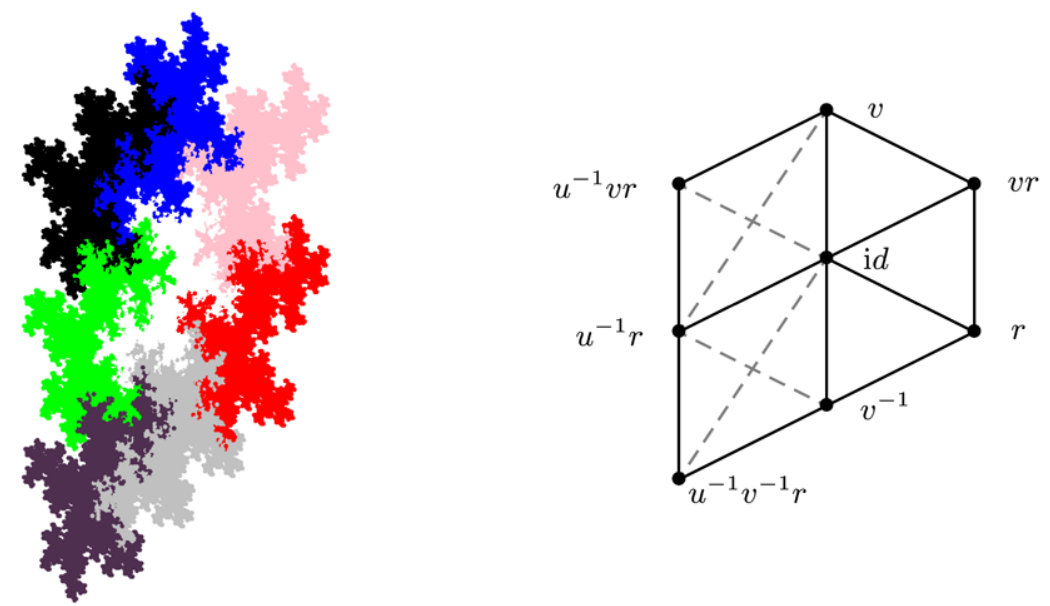

Fig. 10 Disk-like $p 2$-crystile (white) with its seven neighbors
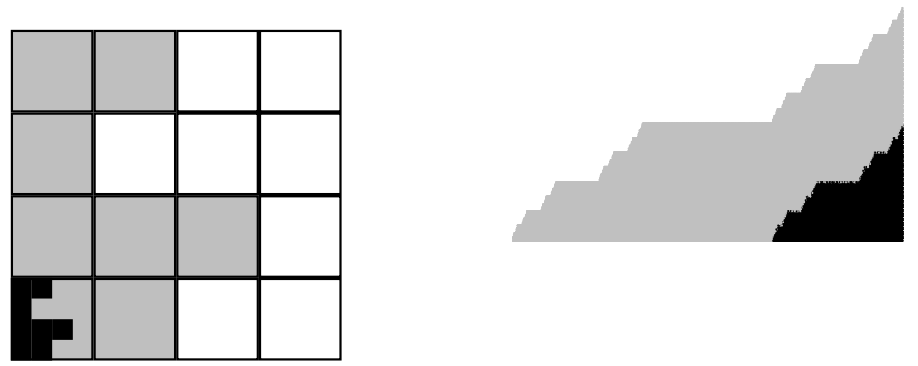

Fig. 11 Disk-like $p$ 2-crystiles having eight neighbors (shape 1) and twelve neighbors
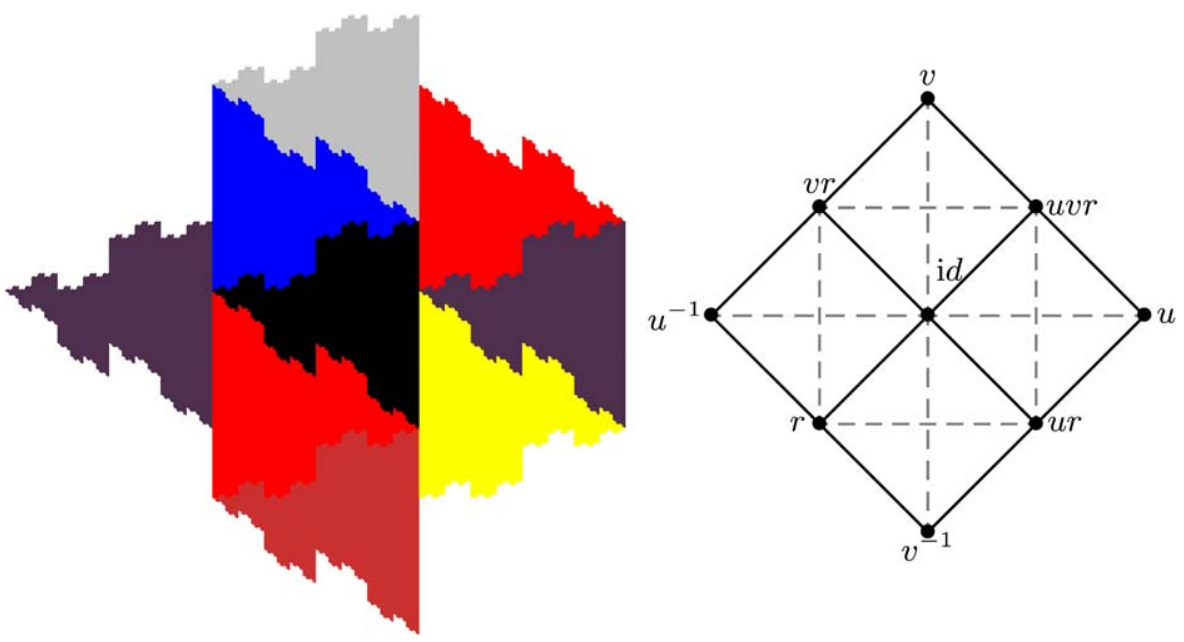

Fig. 12 Disk-like $p 2$-crystile with its eight neighbors (shape 2) 

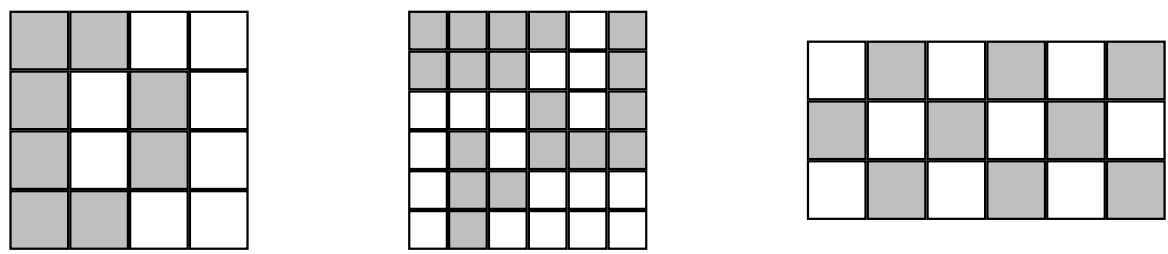

Fig. 13 From left to right: non-disk-like $p 2$-crystiles having eight, nine, and eleven neighbors

12-Neighbor Case Like a right-angled isosceles triangle, the "stair cage" crystile on the right of Fig. 11 gives rise to a $p 2$-tiling where each tile has twelve neighbors. Here, the expansion map is $g(x, y)=(3 x, 2 y)$, and the digit set is $\mathcal{D}=\left\{\mathrm{id}, a_{1}, a_{1}^{2}, b_{1}, a_{1} b_{1} c_{1}, a_{1}^{2} b_{1}^{2}\right\}$ with $a_{1}(x, y)=(x-2, y), b_{1}(x, y)=(x, y+1)$, and $c_{1}(x, y)=(-x,-y)$. It is disk-like by Theorem 2.4(4) (take $a=b_{1}^{-1}, b=a_{1}^{-1} b_{1}$, $\left.c=a_{1} c_{1}\right)$.

\subsection{Examples of Non-disk-Like $p 2$-Crystiles}

We are now moving to the non-disk-like examples, varying the number of neighbors. Since we are interested in connected tiles, the case of six neighbors can be excluded (see our later comments). We already gave Gelbrich's 36- $p 2$-reptile as an example of crystile with seven neighbors. Thus we start with an eight-neighbor example.

Eight-Neighbor Case This example is depicted on Fig. 13. It is a p2-crystile with 16 digits. It is obtained after an obvious modification of the digit set of the disk-like example of Fig. 11; it has the same neighbor set.

Nine-Neighbor Case Again, this tile is constructed from squares (see Fig. 13). It has nine neighbors, among which seven are adjacent.

Ten-Neighbor Case Let $g(x, y)=(-2 x-1 / 2,-x+2 y)$ be the expansion, and let the digit set be $\mathcal{D}=\{\mathrm{id}, u, v, r\}$. As can be seen in Fig. 14, the corresponding crystile $T$ has the neighbor set

$$
\mathcal{S}=\left\{u^{ \pm 1}, v^{ \pm 1},(u v)^{ \pm 1}, r, v r, u^{-1} r, u^{-1} v^{-1} r\right\} .
$$

11-Neighbor Case The crystile of Fig. 13 is trivially non-disk-like and is again a 36-reptile constructed from squares. It has eleven neighbors, and seven of them are adjacent.

12-Neighbor Case Our last example is a perturbation of the stair cage disk-like example. It still has twelve neighbors in the induced tiling and is depicted on Fig. 14. It is obtained by taking the expansion $g(x, y)=(3 x, 3 y)$ and the digit set $\mathcal{D}=$ $\left\{\right.$ id, $\left.a, a^{2}, b, b^{2}, c, a c, b c, a b c\right\}$, where $a(x, y)=(x-2, y), b(x, y)=(x, y+1)$, $c(x, y)=(-x,-y)$. 

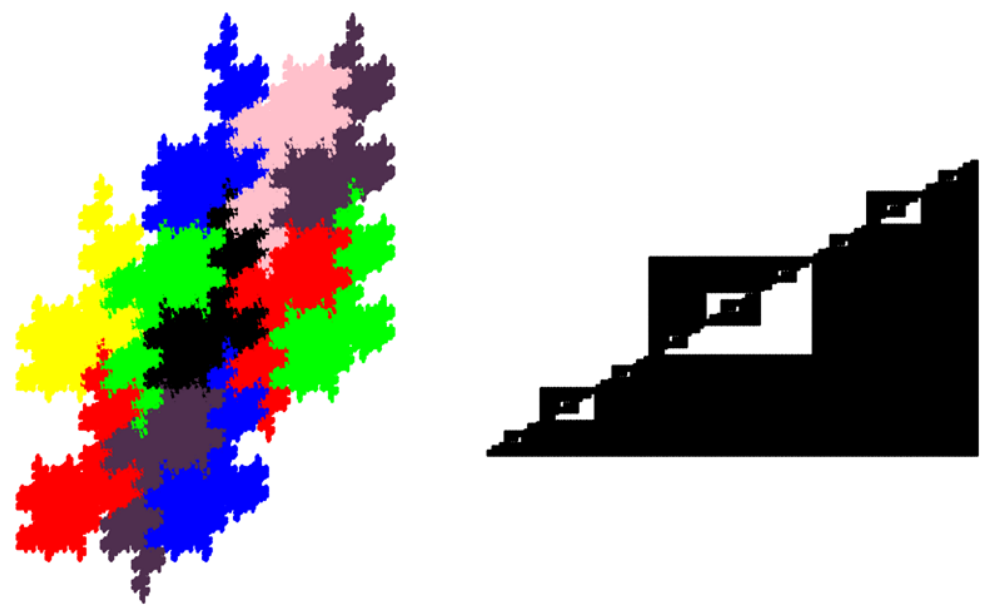

Fig. 14 Non disk-like p2-crystiles having ten neighbors (left) and 12 neighbors (right)

\subsection{Comments and Questions}

We were interested in the neighbor relations in a tiling by crystallographic tiles and reptiles. For $\Gamma$ lattice or $p 2$ group, the minimal neighbor number of a $\Gamma$-tile was shown to be six. In both cases, all neighbors are adjacent. Since a crystile with neighbor set $\mathcal{S}$ is connected if and only if the digit set is $\mathcal{S}$-connected (see [12]), we conclude from Proposition 2.3 and Theorem 2.4 that a lattice or $p 2$-crystile with minimal number of neighbors is connected if and only if it is disk-like. We conjecture that this remains true for the other crystallographic groups for which the least neighbor number is six. Note that not all crystallographic groups have this number as minimal neighbor number, since it is eight for the $\mathrm{pm}$-group (generated by two perpendicular translations and a reflection along one of the translation vectors).

After dealing with the minimal neighbor number, we may now wonder how many neighbors and adjacent neighbors a crystile can have. Indeed, we found in the last section $p 2$-crystiles with six to 12 neighbors, is then every number greater than six admissible? If not, which numbers are forbidden? How are they related to the crystallographic group? Even the lattice case is still open: is every even number greater than six admissible? Some partial results are known for lower number of neighbors and it is also known that lattice reptiles can be constructed with $6+4 k$ neighbors for every $k \in \mathbb{N}$ : these are tiles associated to canonical number systems (see [3]). Concerning the adjacent neighbors, Grünbaum and Shephard's results on normal tilings (see [9]) indicate that 4 and 6 are the only possible numbers of adjacent neighbors for disk-like lattice tiles, and 3, 4, 5, 6 for disk-like $p 2$-tiles, hence these restrictions remain valid for disk-like lattice and $p 2$-crystiles. What about non-disk-like crystiles? We already gave in this study two examples of $p 2$-crystiles with seven adjacent neighbors. Can there be more? Or is this number limited by some rigidity of the crystallographic group?

Acknowledgement We thank the anonymous referee for his valuable suggestions and comments improving the quality of this paper. 


\section{References}

1. Adams, C., Morgan, F., Sullivan, J.M.: When soap bubbles collide. Am. Math. Mon., to appear

2. Akiyama, S., Luo, J., Thuswaldner, J.-M.: On the boundary connectedness of connected tiles. Math. Proc. Camb. Phil. Soc. 137, 397-410 (2004)

3. Akiyama, S., Thuswaldner, J.-M.: The topological structure of fractal tilings generated by quadratic number systems. Comput. Math. Appl. 49, 1439-1485 (2005)

4. Bandt, C., Wang, Y.: Disk-like self-affine tiles in $\mathbb{R}^{2}$. Discrete Comput. Geom. 26, 591-601 (2001)

5. Burckhardt, J.: Die Bewegungsgruppen der Kristallographie. Birkhäuser, Basel (1947)

6. Deng, D.-W., Ngai, S.-Z.: Vertices of self-similar tiles. Ill. J. Math. 49, 857-872 (2005)

7. Engelking, R.: Dimension Theory. PWN/Polish Scientific, Warszawa (1978)

8. Gelbrich, G.: Crystallographic reptiles. Geom. Dedicata 51, 235-256 (1994)

9. Grünbaum, B., Shephard, G.C.: Tilings and Patterns: An Introduction. Freeman, New York (1987)

10. Hata, M.: On the structure of self-similar sets. Jpn. J. Appl. Math. 2, 381-414 (1985)

11. Hutchinson, J.E.: Fractals and self-similarity. Indiana Univ. Math. J. 30, 713-747 (1981)

12. Kirat, I., Lau, K.-S.: On the connectedness of self-affine tiles. J. Lond. Math. Soc. 62, 291-304 (2000)

13. Kuratowski, K.: Topology, vol. II. Academic Press, New York/London (1968)

14. Loridant, B., Luo, J., Thuswaldner, J.: Topology of crystallographic tiles. Geom. Dedicata 128, 113144 (2007)

15. Loridant, B., Luo, J., Thuswaldner, J.: A new criterion for disk-like crystallographic reptiles. Topol. Proc. 31(2), 593-610 (2007)

16. Whyburn, G.T., Duda, E.: Dynamic Topology. Undergraduate Texts in Mathematics. Springer, New York (1979) 